\title{
RAREFIED ELLIPTIC HYPERGEOMETRIC FUNCTIONS
}

\author{
V.P. SPIRIDONOV
}

\begin{abstract}
Two exact evaluation formulae for multiple rarefied elliptic beta integrals related to the simplest lens space are proved. They generalize evaluations of the type I and II elliptic beta integrals attached to the root system $C_{n}$. In a special $n=1$ case, the simplest $p \rightarrow 0$ limit is shown to lead to a new class of $q$-hypergeometric identities. Symmetries of a rarefied elliptic analogue of the Euler-Gauss hypergeometric function are described and the respective generalization of the hypergeometric equation is constructed. Some extensions of the latter function to $C_{n}$ and $A_{n}$ root systems and corresponding symmetry transformations are considered. An application of the rarefied type II $C_{n}$ elliptic hypergeometric function to some eigenvalue problems is briefly discussed.
\end{abstract}

Key words: elliptic hypergeometric functions, elliptic functions, elliptic hypergeometric equation, root systems

\section{Contents}

1. Introduction

2. The elliptic beta integral

3. The rarefied elliptic gamma function

4. A rarefied elliptic beta integral

5. A $C_{n}$ rarefied elliptic beta integral of type I

6. A special $p \rightarrow 0$ limit

7. A $C_{n}$ rarefied elliptic beta integral of type II

8. An analogue of the Euler-Gauss hypergeometric function

9. Extension of the multiple integral symmetry transformations

10. Conclusion

References

To the memory of G. M. Vereshkov, my teacher

\section{InTRODUCTION}

Hypergeometric functions are central objects in the theory of special functions [2]. Elliptic functions (i.e., meromorphic doubly-periodic functions) form another key family from this world. Nowadays it is known that these two classical sets of functions are deeply tied to each other. Frenkel and Turaev [20] investigated elliptic functions that appeared as solutions of the Yang-Baxter equation in [10] and have shown that they 
have the form resembling hypergeometric series and obey similar properties. This has led to the discovery of remarkable terminating elliptic hypergeometric series summation and transformation formulae (infinite series of such type do not converge) generalizing the corresponding Jackson sum and Bailey transformation [2, 25]. The biorthogonal functions expressed in terms of such series have been constructed in [54] (discrete measure) and [44] (continuous measure).

The genuine elliptic hypergeometric functions which are transcendental over the field of elliptic functions were discovered in [43]. They are determined by a specific class of integrals whose integrands satisfy linear $q$-difference equations with $p$-elliptic coefficients, and this property can be used for a general definition of such functions [44]. Elliptic hypergeometric integrals can be reduced to elliptic hypergeometric series (particular elliptic functions) through residues calculus, to general class of $q$-hypergeometric functions (by taking a simple limit $p \rightarrow 0$ [43], or by more complicated degenerations [35]) and to ordinary hypergeometric functions. Unification of elliptic and hypergeometric insights elucidated various previously known properties of the corresponding functions. For instance, it explained the origin of hypergeometric notions of well-poisedness, very-well-poisedness and balancing in terms of the ellipticity conditions [49]. The unique nature of the most interesting elliptic hypergeometric functions is established by their symmetries associated with two independent elliptic curves and two independent root systems, or compact Lie groups (one attached to the Haar measure defining multiple integrals and another one living in the space of free parameters of these functions). The basics of the theory of elliptic hypergeometric functions is surveyed in [49], a more recent review is given in [40].

The elliptic beta integral [43] is until now the only known computable integral among univariate elliptic hypergeometric integrals. Its evaluation formula (see identity (9)) can be interpreted as an elliptic analogue of Newton's binomial theorem. Moreover, this integral represents a top known generalization of Euler's beta function and serves as the biorthogonality measure for particular elliptic functions (actually, the product of two elliptic functions with different nomes [44]) forming the most general set of special functions extending the Jacobi and Askey-Wilson polynomials [2], etc. It has found remarkable applications in theoretical physics, the first one being in quantum mechanical eigenvalue problems [46, 48]. The most important physical interpretation of formula (9) was discovered by Dolan and Osborn in quantum field theory [17] - it proves the equality of superconformal indices of two nontrivial four dimensional supersymmetric models connected by the Seiberg duality. Currently this gives the most rigorous mathematical confirmation of the confinement phenomenon.

The next important representative of univariate elliptic hypergeometric integrals is an elliptic analogue of the Euler-Gauss hypergeometric function. It contains two more free parameters than the elliptic beta integral and satisfies an elliptic hypergeometric equation [46, 48]. It appeared for the first time in [44] together with a nontrivial symmetry transformation related to the exceptional root system $E_{7}$ (an identification with the corresponding Weyl group action was established in [36]). 
Another line of generalizations of the elliptic beta integral considers multiple integrals. Here, the very first multiple elliptic beta integrals were defined by van Diejen and the present author [13, 14] in relation to the root system $C_{n}$. The integrals defined in [14] are referred to as of type I, and those of [13] as of type II. The latter integral is a generalization of the Selberg integral [42, which follows from its simple $p \rightarrow 0$ reduction to a Gustafson integral with previously known reductions [26, 27]. The importance of Selberg's multiple beta integral and its generalizations is surveyed in [19]. The classification of integrals as type I or II is inspired by differences in the methods used for proving corresponding exact evaluation formulae and in the number of free parameters contained in them (which depends on the rank of the root system for type I and is fixed for type II integrals). The type II integral evaluation was proven to follow from the type I integral identity as a result of simple, purely algebraic considerations [14. However, the type I integral evaluation was not completely proven in [14, it depended on a vanishing condition of a certain integral. The first complete proof of this integration formula was given by Rains in [36], and shortly thereafter the present author found an elementary proof of the type I integral evaluations [47] (this method was used also for considering other integrals in [53] and we employ it here as well). Nowadays, the number of known elliptic hypergeometric integrals admitting (proven or conjectural) either exact evaluation or a nontrivial symmetry transformation described by the Weyl groups of various root systems is very large, see, e.g. [8, 9, 36, 49, 50, 51, 53. In this paper we crucially follow the logic established in [13, 14] and use it for a derivation of elliptic hypergeometric identities of a new type.

The discovery of relations between superconformal indices and elliptic hypergeometric functions [17] has attracted much attention. In addition to its systematic consideration in [50, 51], which resulted in the formulation of very many new mathematical conjectures and the discovery of new physical Seiberg dualities, there have been other important developments. For instance, such integrals emerged in two-dimensional topological field theories [22], their properties describe symmetry enhancement phenomena [15], the elliptic Fourier transform introduced in [45] in the rank 1 case and extended to arbitrary rank root systems in 53] plays an important role in the discussion of five dimensional duality questions [24], etc. For a recent survey of this subject, see [38].

In this setting, the standard elliptic hypergeometric integrals are related to the Hopf manifold $S^{1} \times S^{3}$, which plays a role of compact space-time for the corresponding four dimensional superconformal field theories. However, this is only one of many admissible four dimensional manifolds for which one can compute superconformal indices. The next level of topological complication is related to the replacement of the $S^{3}$-factor by the lens space. It was considered first in [7], where an analogue of the elliptic gamma function for the simplest lens space was introduced. Some further essential developments of this subject can be found in [39].

Recently, Kels [31] proposed an extension of the univariate elliptic beta integral associated with the simplest lens space. It involves some additional discrete variables and a replacement of the single integration by a finite sum of integrations. Earlier examples of 
similar sums of simpler integrals can be found in [6, 30, 33. In particular, they emerge already in the representation theory of $\operatorname{SL}(2, \mathbb{C})$ group [33. In this work we consider elliptic hypergeometric functions associated with the lens space by a different method and confirm the result derived in [31]. Moreover, we find another similar extension of formula (9) and show that it has a highly nontrivial $p \rightarrow 0$ limit leading to a new type of $q$-hypergeometric identities. Furthermore, we propose two explicit multiple hybrid sumintegrals generalizing the type I and II elliptic beta integrals of [13, 14]. It will be shown that the general class of such functions, which we call "rarefied elliptic hypergeometric functions", matches with the general definition of elliptic hypergeometric functions of [44 when it is applied to the case of sums of integrals. As follows from their structure, these functions should be considered as $2 n$-variate "sum-integral" objects. One set of $n$ variables is discrete and defines the $n$-tuple summation and another set of $n$ continuous variables defines the $n$-tuple integration.

We define a rarefied elliptic analogue of the Euler-Gauss hypergeometric function and construct the corresponding $W\left(E_{7}\right)$ symmetry transformations and elliptic hypergeometric equation. The type II extension of the latter function to the root systems $C_{n}$ is proposed and an extension of Rains' transformation [36] is conjectured. An application of this function to the eigenvalue problem for a particular finite-difference operator is briefly discussed. We consider also symmetry transformations for the rarefied multiple elliptic hypergeometric functions of type I on the root systems $C_{n}$ and $A_{n}$. In the concluding section we outline some prospects for further development of the derived results.

\section{The ELliptic Beta INTEGRAL}

In this section we describe particular elliptic hypergeometric integrals introduced in [13, 14, 43]. For $p \in \mathbb{C},|p|<1$, we define the infinite product

$$
(z ; p)_{\infty}=\prod_{j=0}^{\infty}\left(1-z p^{j}\right), \quad z \in \mathbb{C} .
$$

The theta function

$$
\theta(z ; p)=(z ; p)_{\infty}\left(p z^{-1} ; p\right)_{\infty}, \quad z \in \mathbb{C}^{*},
$$

obeys the following symmetry properties

$$
\theta\left(x^{-1} ; p\right)=\theta(p x ; p)=-x^{-1} \theta(x ; p) .
$$

We shall need the general quasiperiodicity relation

$$
\theta\left(p^{k} z ; p\right)=(-z)^{-k} p^{-\frac{k(k-1)}{2}} \theta(z ; p), \quad k \in \mathbb{Z} .
$$

The "addition law" for theta functions has the form

$$
\theta\left(x w^{ \pm 1}, y z^{ \pm 1} ; p\right)-\theta\left(x z^{ \pm 1}, y w^{ \pm 1} ; p\right)=y w^{-1} \theta\left(x y^{ \pm 1}, w z^{ \pm 1} ; p\right),
$$


where $x, y, w, z \in \mathbb{C}^{*}$. We use the convention

$$
\theta\left(x_{1}, \ldots, x_{k} ; p\right)=\prod_{j=1}^{k} \theta\left(x_{j} ; p\right), \quad \theta\left(t x^{ \pm 1} ; p\right)=\theta\left(t x, t x^{-1} ; p\right) .
$$

For arbitrary $q \in \mathbb{C}$ and $n \in \mathbb{Z}$, the elliptic Pochhammer symbol is defined as

$$
\theta(x ; p \mid q)_{n}:= \begin{cases}\prod_{j=0}^{n-1} \theta\left(x q^{j} ; p\right), & \text { for } n>0 \\ \prod_{j=1}^{-n} \theta\left(x q^{-j} ; p\right)^{-1}, & \text { for } n<0\end{cases}
$$

and $\theta(x ; p \mid q)_{0}=1$.

The first order $q$-difference equation

$$
f(q z ; p, q)=\theta(z ; p) f(z ; p, q), \quad q \in \mathbb{C}^{*},
$$

has a particular solution

$$
f(z ; p, q)=\Gamma(z ; p, q):=\prod_{j, k=0}^{\infty} \frac{1-z^{-1} p^{j+1} q^{k+1}}{1-z p^{j} q^{k}}, \quad|p|,|q|<1, \quad z \in \mathbb{C}^{*},
$$

called the (standard) elliptic gamma function. Note that equation (3) does not require $|q|<1$, whereas solution (44) is restricted to this domain.

The function $\Gamma(z ; p, q)$ is a building block for elliptic hypergeometric integrals with an interesting history. The problem of generalizing Euler's gamma function to a similar function "of the second order" was considered by Alexeevski long ago [1]. Later Barnes derived similar results, but he went further and defined multiple gamma functions of arbitrary order [4]. Jackson [28] considered this problem from a different angle and investigated the well-known $q$-gamma function and defined the double $(p, q)$-gamma function. In the same work he constructed the elliptic gamma function with equal periods $p=q$ and indicated how to construct the general case, however, his results did not attract the deserved attention. This function was implicitly discovered also by Baxter in [5], since the partition function of the eight-vertex model is given by a particular combination of four such functions (see [18 for an explicit relation). More recently function (44) was considered by Ruijsenaars in [41], where the term "elliptic gamma function" was introduced. A systematic investigation of this function was performed by Felder and Varchenko [18] who discovered its $\mathrm{SL}(3, \mathbb{Z})$ symmetry transformations and gave a cohomological interpretation. In [44 the author constructed the modified elliptic gamma function, which gives a solution of equation (3) in the regime $|q|=1$ (it is meromorphic in $\log z$, not $z$ ) and has nice physical applications [52]. In [21], Friedman and Ruijsenaars explicitly expressed the standard elliptic gamma function (44) as a particular combination of four Barnes gamma functions of the third order (such a relation was suggested also earlier in [44 up to the exponential of a Bernoulli polynomial factor).

The elliptic gamma function (4) has the following properties

$$
\begin{gathered}
\Gamma(z ; p, q)=\Gamma(z ; q, p), \\
\Gamma(q z ; p, q)=\theta(z ; p) \Gamma(z ; p, q), \quad \Gamma(p z ; p, q)=\theta(z ; q) \Gamma(z ; p, q),
\end{gathered}
$$




$$
\begin{gathered}
\Gamma(z ; p, q) \Gamma\left(\frac{p q}{z} ; p, q\right)=1 \\
\Gamma(\sqrt{p q} ; p, q)=1 \\
\lim _{z \rightarrow 1}(1-z) \Gamma(z ; p, q)=\frac{1}{(p ; p)_{\infty}(q ; q)_{\infty}} .
\end{gathered}
$$

Its poles and zeros form the double base geometric progressions

$$
z_{\text {poles }}=p^{-j} q^{-k}, \quad z_{\text {zeros }}=p^{j+1} q^{k+1}, \quad j, k \in \mathbb{Z}_{\geq 0} .
$$

The elliptic Pochhammer symbol can be written in the form

$$
\theta(z ; p \mid q)_{m}=\frac{\Gamma\left(z q^{m} ; p, q\right)}{\Gamma(z ; p, q)}, \quad m \in \mathbb{Z} .
$$

We also define the elliptic gamma function of the second order

$$
\Gamma(z ; p, q, t)=\prod_{j, k, l=0}^{\infty}\left(1-z p^{j} q^{k} t^{l}\right)\left(1-z^{-1} p^{j+1} q^{k+1} t^{l+1}\right), \quad|t|,|p|,|q|<1, z \in \mathbb{C}^{*} .
$$

It satisfies the equation

$$
\Gamma(q z ; p, q, t)=\Gamma(z ; p, t) \Gamma(z ; p, q, t)
$$

and its partners obtained by permutation of the bases $p, q, t$ and the inversion relation

$$
\Gamma(p q t z ; p, q, t)=\Gamma\left(z^{-1} ; p, q, t\right) .
$$

The elliptic beta integral evaluation formula, which serves as a basis for the whole general theory of elliptic hypergeometric integrals, has the following form [43].

Theorem 1. Let $t_{1}, \ldots, t_{6}, p, q \in \mathbb{C}^{*}$ such that $\left|t_{a}\right|,|p|,|q|<1$ and $\prod_{a=1}^{6} t_{a}=p q$. Then

$$
\frac{(p ; p)_{\infty}(q ; q)_{\infty}}{2} \int_{\mathbb{T}} \frac{\prod_{a=1}^{6} \Gamma\left(t_{a} z^{ \pm 1} ; p, q\right)}{\Gamma\left(z^{ \pm 2} ; p, q\right)} \frac{d z}{2 \pi \mathrm{i} z}=\prod_{1 \leq a<b \leq 6} \Gamma\left(t_{a} t_{b} ; p, q\right)
$$

where $\mathbb{T}$ is the positively oriented unit circle.

Here we use the compact notation

$$
\begin{aligned}
& \Gamma\left(t_{1}, \ldots, t_{n} ; p, q\right):=\Gamma\left(t_{1} ; p, q\right) \ldots \Gamma\left(t_{n} ; p, q\right), \quad \Gamma\left(t z^{ \pm k} ; p, q\right):=\Gamma\left(t z^{k} ; p, q\right) \Gamma\left(t z^{-k} ; p, q\right) \\
& \Gamma\left(z^{ \pm 1} w^{ \pm 1} ; p, q\right):=\Gamma(z w ; p, q) \Gamma\left(z^{-1} w ; p, q\right) \Gamma\left(z w^{-1} ; p, q\right) \Gamma\left(z^{-1} w^{-1} ; p, q\right) .
\end{aligned}
$$

If one substitutes in (9) $t_{6}=p q / \prod_{a=1}^{5} t_{a}$, uses the inversion formula (5), and takes the limit $p \rightarrow 0$ for fixed $t_{1}, \ldots, t_{5}$ and $q$, then one obtains the Rahman $q$-beta integral [34] (see formula (65) below for $r=1$ ).

In [13, 14], van Diejen and the present author proposed two multiple generalizations of the integration formula (9) in relation with the root system $C_{n}$. The type I integral 
has the following form. Let $z_{1}, \ldots, z_{n} \in \mathbb{T}$ and complex parameters $t_{1}, \ldots, t_{2 n+4}$ and $p, q$ satisfy the constraints $|p|,|q|,\left|t_{a}\right|<1$ and $\prod_{j=1}^{2 n+4} t_{a}=p q$. Then

$$
\begin{aligned}
\kappa_{n} \int_{\mathbb{T}^{n}} \prod_{1 \leq j<k \leq n} \frac{1}{\Gamma\left(z_{j}^{ \pm 1} z_{k}^{ \pm 1} ; p, q\right)} \prod_{j=1}^{n} \frac{\prod_{a=1}^{2 n+4} \Gamma\left(t_{a} z_{j}^{ \pm 1} ; p, q\right)}{\Gamma\left(z_{j}^{ \pm 2} ; p, q\right)} \prod_{j=1}^{n} \frac{d z_{j}}{z_{j}} \\
=\prod_{1 \leq a<b \leq 2 n+4} \Gamma\left(t_{a} t_{b} ; p, q\right), \quad \kappa_{n}=\frac{(p ; p)_{\infty}^{n}(q ; q)_{\infty}^{n}}{(4 \pi \mathrm{i})^{n} n !} .
\end{aligned}
$$

This integral evaluation can be considered as a high level generalization of a Dixon identity [16].

The type II $C_{n}$-integral has a structurally different form. Let complex parameters $t, t_{a}(a=1, \ldots, 6), p$, and $q$ satisfy the conditions $|p|,|q|,|t|,\left|t_{a}\right|<1$, and $t^{2 n-2} \prod_{a=1}^{6} t_{a}=$ $p q$. Then

$$
\begin{gathered}
\kappa_{n} \int_{\mathbb{T}^{n}} \prod_{1 \leq j<k \leq n} \frac{\Gamma\left(t z_{j}^{ \pm 1} z_{k}^{ \pm 1} ; p, q\right)}{\Gamma\left(z_{j}^{ \pm 1} z_{k}^{ \pm 1} ; p, q\right)} \prod_{j=1}^{n} \frac{\prod_{a=1}^{6} \Gamma\left(t_{a} z_{j}^{ \pm 1} ; p, q\right)}{\Gamma\left(z_{j}^{ \pm 2} ; p, q\right)} \prod_{j=1}^{n} \frac{d z_{j}}{z_{j}} \\
=\prod_{j=1}^{n}\left(\frac{\Gamma\left(t^{j} ; p, q\right)}{\Gamma(t ; p, q)} \prod_{1 \leq a<b \leq 6} \Gamma\left(t^{j-1} t_{a} t_{b} ; p, q\right)\right) .
\end{gathered}
$$

The latter integral can be interpreted as an elliptic extension of the Selberg integral [2, 19, 42]. For $n=1$ both these multiple integrals reduce to the elliptic beta integral (9). In the simplest $p \rightarrow 0$ limit, similar to the mentioned reduction to the Rahman integral, one reproduces the Gustafson $C_{n}$-integrals from [27].

\section{The RAREFIED ELLIPTIC GAMMA FUNCTION}

An analogue of the elliptic gamma function for the simplest lens space was introduced in [7]. Some functions involving it were considered in [31, 39]. In comparison to the standard elliptic hypergeometric integrals, they contain some integer parameters and involve finite summations over discrete variables additional to the standard integrations. In this work we use the analysis of [7, 31, 39] as an inspiration for considering the general structure of elliptic hypergeometric functions of such type, which we call the rarefied elliptic hypergeometric functions.

The lens space elliptic gamma function is determined by a particular product of two standard elliptic gamma functions with different bases

$$
\begin{aligned}
\gamma^{(r)}(z, m ; p, q) & :=\Gamma\left(z p^{m} ; p^{r}, p q\right) \Gamma\left(z q^{r-m} ; q^{r}, p q\right) \\
& =\prod_{j, k=0}^{\infty} \frac{1-z^{-1} p^{-m}(p q)^{j+1} p^{r(k+1)}}{1-z p^{m}(p q)^{j} p^{r k}} \frac{1-z^{-1} q^{m}(p q)^{j+1} q^{r k}}{1-z q^{r-m}(p q)^{j} q^{r k}}
\end{aligned}
$$

which, in addition to the variable $z \in \mathbb{C}^{*}$, involves two integers $r \in \mathbb{Z}_{>0}$ and $m \in \mathbb{Z}$. It has poles at the points

$$
z_{\text {poles }}=p^{-m-j-r k} q^{-j}, p^{-j} q^{m-r-j-r k}
$$


and zeros

$$
z_{\text {zeros }}=p^{j+1+r(k+1)-m} q^{j+1}, p^{j+1} q^{m+j+1+r k}, \quad j, k \in \mathbb{Z}_{\geq 0} .
$$

According to [7], this function is associated with the superconformal index of a chiral superfield on the space-time $S^{1} \times L(r, k)$ with $k=-1$, where $L(r, k)$ is the lens space defined by the identification of points $\left(e^{2 \pi \mathrm{i} / r} z_{1}, e^{2 \pi \mathrm{i} k / r} z_{2}\right) \sim\left(z_{1}, z_{2}\right)$ in the complex representation of the $S^{3}$-sphere, $\left|z_{1}^{2}\right|+\left|z_{2}\right|^{2}=1$. Erroneously, in the physics literature the same space is denoted as $L(r,-k)$, which should not be confused with our notation. To the present time, the superconformal indices have only been computed for the space $L(r,-1)$. It is worth of mentioning that the manifolds $L(r,-1)$ (or $L(r, r-1)$ ) and $L(r, 1)$ are homeomorphic and differ by orientation only. At the moment it is not clear whether the rarefied elliptic hypergeometric functions (superconformal indices) described below can distinguish them or not.

The function $\gamma^{(r)}(z, m ; p, q)$ looks rather different from $\Gamma(z ; p, q)$, since it involves three bases $p^{r}, q^{r}, p q$ and the discrete variable $m$. Let us show that, in fact, it is nothing but a special product of standard elliptic gamma functions with bases $p^{r}$ and $q^{r}$. Consider the double elliptic gamma function $\Gamma(z ; p, q, t)$ with a special choice of the third base parameter $t=p q$. With its help, we can write

$$
\begin{aligned}
\gamma^{(r)}(z, m ; p, q) & =\frac{\Gamma\left(q^{r} z p^{m} ; p^{r}, q^{r}, p q\right)}{\Gamma\left(z p^{m} ; p^{r}, q^{r}, p q\right)} \frac{\Gamma\left(p^{r} z q^{r-m} ; p^{r}, q^{r}, p q\right)}{\Gamma\left(z q^{r-m} ; p^{r}, q^{r}, p q\right)} \\
& =\frac{\Gamma\left((p q)^{m} q^{r-m} z ; p^{r}, q^{r}, p q\right)}{\Gamma\left(q^{r-m} z ; p^{r}, q^{r}, p q\right)} \frac{\Gamma\left((p)^{r-m} p^{m} z ; p^{r}, q^{r}, p q\right)}{\Gamma\left(p^{m} z ; p^{r}, q^{r}, p q\right)} .
\end{aligned}
$$

Using equation (8), from the latter relation we derive the product of two Pochhammertype symbols built out of the elliptic gamma function with the bases $p^{r}$ and $q^{r}$. For $0 \leq m \leq r$ we obtain the expression

$$
\gamma^{(r)}(z, m ; p, q)=\prod_{k=0}^{m-1} \Gamma\left(q^{r-m} z(p q)^{k} ; p^{r}, q^{r}\right) \prod_{k=0}^{r-m-1} \Gamma\left(p^{m} z(p q)^{k} ; p^{r}, q^{r}\right)
$$

for $m<0$ we have

$$
\gamma^{(r)}(z, m ; p, q)=\frac{\prod_{k=0}^{r-m-1} \Gamma\left(p^{m} z(p q)^{k} ; p^{r}, q^{r}\right)}{\prod_{k=1}^{-m} \Gamma\left(q^{r-m} z(p q)^{-k} ; p^{r}, q^{r}\right)}
$$

and for $m>r$,

$$
\gamma^{(r)}(z, m ; p, q)=\frac{\prod_{k=0}^{m-1} \Gamma\left(q^{r-m} z(p q)^{k} ; p^{r}, q^{r}\right)}{\prod_{k=1}^{m-r} \Gamma\left(p^{m} z(p q)^{-k} ; p^{r}, q^{r}\right)} .
$$

The second order elliptic gamma function is related to superconformal indices of six dimensional field theories. Therefore it is natural to expect that there exists some physical meaning of the function (15) from the point of view of compactification of six dimensional theories to the lens space [7, 39]. 
From (16) it follows that for $r=1, m=0$ and $r=1, m=1$ we have the standard elliptic gamma function

$$
\gamma^{(1)}(z, 0 ; p, q)=\gamma^{(1)}(z, 1 ; p, q)=\Gamma(z ; p, q) .
$$

These equalities are related to the following factorized representation of the elliptic gamma function

$$
\Gamma(z ; p, q)=\prod_{j=0}^{\infty} \frac{1-z^{-1}(p q)^{j+1}}{1-z(p q)^{j}} \prod_{j, k=0}^{\infty} \frac{1-z^{-1}(p q)^{j+1} p^{k+1}}{1-z(p q)^{j} p^{k+1}} \frac{1-z^{-1}(p q)^{j+1} q^{k+1}}{1-z(p q)^{j} q^{k+1}} .
$$

For $r=1$ and $m \neq 0$ one can deduce directly from the definition (12) the recurrence relation

$$
\gamma^{(1)}(z, m+1 ; p, q)=\frac{\theta\left(z p^{m} ; p q\right)}{\theta\left(z q^{-m} ; p q\right)} \gamma^{(1)}(z, m ; p, q)
$$

yielding

$$
\begin{aligned}
\gamma^{(1)}(z, m ; p, q) & =\theta(z ; p q \mid p)_{m} \theta(q z ; p q \mid q)_{-m} \Gamma(z ; p, q) \\
& =\left(-\frac{\sqrt{p q}}{z}\right)^{\frac{m(m-1)}{2}}\left(\frac{q}{p}\right)^{\frac{m(m-1)(2 m-1)}{12}} \Gamma(z ; p, q), \quad m \in \mathbb{Z} .
\end{aligned}
$$

As a result, the normalization condition $\Gamma(\sqrt{p q} ; p, q)=1$ is replaced by a more complicated relation

$$
\gamma^{(1)}(\sqrt{p q}, m ; p, q)=(-1)^{\frac{m(m-1)}{2}}\left(\frac{q}{p}\right)^{\frac{m(m-1)(2 m-1)}{12}} .
$$

Instead of the exact $(p, q)$-permutation symmetry one now has

$$
\gamma^{(r)}(z, m ; p, q)=\gamma^{(r)}(z, r-m ; q, p) .
$$

The $\gamma^{(r)}$-function has an important quasiperiodicity property

$$
\begin{aligned}
& \frac{\gamma^{(r)}(z, m+k r ; p, q)}{\gamma^{(r)}(z, m ; p, q)}=\prod_{l=0}^{k-1} \frac{\theta\left(z p^{m+l r} ; p q\right)}{\theta\left(z q^{-m-l r} ; p q\right)} \\
& =\left(-\frac{\sqrt{p q}}{z}\right)^{m k+r \frac{k(k-1)}{2}}\left(\frac{q}{p}\right)^{k\left(\frac{1}{2} m^{2}+m r \frac{k-1}{2}+r^{2} \frac{(k-1)(2 k-1)}{12}\right)}, \quad k \in \mathbb{Z} .
\end{aligned}
$$

Since any integer $m$ can be represented in the form $l+k r$ with $0 \leq l \leq r-1, k \in \mathbb{Z}$, formulae (16) and (21) provide general representation of the $\gamma^{(r)}(z, m ; p, q)$-function as a product of elliptic gamma functions with the bases $p^{r}$ and $q^{r}$ up to some (cumbersome, but elementary) exponential factor.

The inversion relation has the form

$$
\gamma^{(r)}(z, m ; p, q) \gamma^{(r)}\left(\frac{p q}{z}, r-m ; p, q\right)=1,
$$


which is proved by using the definition (12) and formula (5). The most elementary equations for this function have the form

$$
\begin{aligned}
& \gamma^{(r)}(q z, m+1 ; p, q)=\theta\left(z p^{m} ; p^{r}\right) \gamma^{(r)}(z, m ; p, q), \\
& \gamma^{(r)}(p z, m-1 ; p, q)=\theta\left(z q^{r-m} ; q^{r}\right) \gamma^{(r)}(z, m ; p, q) .
\end{aligned}
$$

Let us normalize the $\gamma^{(r)}(z, m ; p, q)$-function as follows

$$
\Gamma^{(r)}(z, m ; p, q):=\left(-\frac{z}{\sqrt{p q}}\right)^{\frac{m(m-1)}{2}}\left(\frac{p}{q}\right)^{\frac{m(m-1)(2 m-1)}{12}} \gamma^{(r)}(z, m ; p, q) .
$$

We call this function the rarefied elliptic gamma function. Its poles and zeros lie at the same points as in (13) and (14). For $r=1$, independently of the value of $m \in \mathbb{Z}$, one has the equality

$$
\Gamma^{(1)}(z, m ; p, q)=\Gamma(z ; p, q),
$$

which is the source for the normalizing multiplier choice in (24).

The discrete variable quasiperiodicity takes now the form

$$
\frac{\Gamma^{(r)}(z, m+k r ; p, q)}{\Gamma^{(r)}(z, m ; p, q)}=\left[\left(-\frac{z}{\sqrt{p q}}\right)^{2 m+r k}\left(\frac{p}{q}\right)^{m(m+r k)+\frac{r\left(2 r k^{2}-1\right)}{6}}\right]^{\frac{1}{2} k(r-1)}, \quad k \in \mathbb{Z} .
$$

For this function the permutation of $p$ and $q$ is equivalent to the change of sign $m \rightarrow-m$,

$$
\Gamma^{(r)}(z, m ; p, q)=\Gamma^{(r)}(z,-m ; q, p) .
$$

The inversion relation also takes a natural compact form

$$
\Gamma^{(r)}(z, m ; p, q) \Gamma^{(r)}\left(\frac{p q}{z},-m ; p, q\right)=1 .
$$

As a consequence one has the following relations

$$
\Gamma^{(r)}(\sqrt{p q}, m ; p, q) \Gamma^{(r)}(\sqrt{p q},-m ; p, q)=1
$$

and

$$
\Gamma^{(r)}(\sqrt{p q}, 0 ; p, q)=1
$$

which can be used as a normalization condition. For computing the residues we shall need the following limiting relation

$$
\lim _{z \rightarrow 1}(1-z) \Gamma^{(r)}(z, 0 ; p, q)=\lim _{z \rightarrow 1}(1-z) \gamma^{(r)}(z, 0 ; p, q)=\frac{1}{\left(p^{r} ; p^{r}\right)_{\infty}\left(q^{r} ; q^{r}\right)_{\infty}}
$$

which is easily established from the representation (16), relation (6), and the identity $\prod_{k=1}^{r-1} \Gamma\left((p q)^{k} ; p^{r}, q^{r}\right)=1$.

The elementary recurrence relations take the form

$$
\begin{aligned}
& \Gamma^{(r)}(q z, m+1 ; p, q)=(-z)^{m} p^{\frac{m(m-1)}{2}} \theta\left(z p^{m} ; p^{r}\right) \Gamma^{(r)}(z, m ; p, q), \\
& \Gamma^{(r)}(p z, m-1 ; p, q)=(-z)^{-m} q^{\frac{m(m+1)}{2}} \theta\left(z q^{-m} ; q^{r}\right) \Gamma^{(r)}(z, m ; p, q) .
\end{aligned}
$$


The second equality is equivalent to the first one, but in the explicit computations both forms are equally heavily used, as well as the following relations

$$
\begin{aligned}
& \Gamma^{(r)}\left(q^{-1} z, m-1 ; p, q\right)=\left(-\frac{z}{p q}\right)^{-m} p^{-\frac{m(m+1)}{2}} \frac{\Gamma^{(r)}(z, m ; p, q)}{\theta\left(z^{-1} q p^{1-m} ; p^{r}\right)} \\
& \Gamma^{(r)}\left(p^{-1} z, m+1 ; p, q\right)=\left(-\frac{z}{p q}\right)^{m} q^{-\frac{m(m-1)}{2}} \frac{\Gamma^{(r)}(z, m ; p, q)}{\theta\left(z^{-1} p q^{1+m} ; q^{r}\right)} .
\end{aligned}
$$

Note that equations (30) do not determine uniquely the function $\Gamma^{(r)}(z, m ; p, q)$. The general solution of these equations has the form $\Gamma^{(r)}(z, m ; p, q) \varphi_{m}(z)$, where the functions $\varphi_{m}(z)$ satisfy the recurrences

$$
\varphi_{m+1}(q z)=\varphi_{m}(z), \quad \varphi_{m-1}(p z)=\varphi_{m}(z) .
$$

Resolution of the first equation yields $\varphi_{m}(z)=\varphi_{0}\left(q^{-m} z\right)$ for arbitrary function $\varphi_{0}(z)$. The second equation yields $\varphi_{0}(p q z)=\varphi_{0}(z)$, i.e. $\varphi_{0}(z)$ is an elliptic function of $z$ with the modular parameter $p q$,

$$
\varphi_{0}(z)=\prod_{k=1}^{K} \frac{\theta\left(\alpha_{k} z ; p q\right)}{\theta\left(\beta_{k} z ; p q\right)}, \quad \prod_{k=1}^{K} \alpha_{k}=\prod_{k=1}^{K} \beta_{k},
$$

for some integer $K=0,2,3, \ldots$ (the order of this elliptic function). Here the parameters $\alpha_{k}, \beta_{k} \in \mathbb{C}^{*}$ are arbitrary (up to one constraint) variables forming the divisor set of $\varphi_{0}(z)$. So, the space of solutions of interest has a functional freedom. However, the quasiperiodicity condition (25) removes this freedom. Indeed, as a consequence of (25) one gets the additional constraint $\varphi_{0}\left(q^{r} z\right)=\varphi_{0}(z)$. For incommensurate $p$ and $q$, all indicated restrictions for $\varphi_{0}(z)$ can be satisfied only by a constant, $\varphi_{0}(z)=$ const, which is fixed by the normalization condition (28).

For $0 \leq m \leq r$, one can write

$$
\begin{aligned}
\Gamma^{(r)}(z, m ; p, q) & =(-z)^{\frac{m(m-1)}{2}} p^{\frac{m(m-1)(m-2)}{6}} q^{-\frac{m\left(m^{2}-1\right)}{6}} \\
\times & \prod_{k=0}^{m-1} \Gamma\left(q^{r-m} z(p q)^{k} ; p^{r}, q^{r}\right) \prod_{k=0}^{r-m-1} \Gamma\left(p^{m} z(p q)^{k} ; p^{r}, q^{r}\right) .
\end{aligned}
$$

This relation together with the quasiperiodicity (25) expresses the $\Gamma^{(r)}(z, m ; p, q)$-function with arbitrary $m$ as a product of ordinary elliptic gamma functions, since any $m$ can be represented in the form $\ell+k r$ with $0 \leq \ell \leq r-1, k \in \mathbb{Z}$. Therefore it is natural to expect that all interesting integrals constructed from the $\Gamma^{(r)}(z, m ; p, q)$-function can be related to some standard elliptic hypergeometric integrals.

Finally, we stress that the normalization of the $\gamma^{(r)}$-function which we have chosen is not unique. It is easy to find a multiplier $\mu(z, m)$ such that the product $\tilde{\Gamma}^{(r)}(z, m ; p, q):=$ $\mu(z, m) \gamma^{(r)}(z, m ; p, q)$ will be $r$-periodic, $\tilde{\Gamma}^{(r)}(z, m+r ; p, q)=\tilde{\Gamma}^{(r)}(z, m ; p, q)$. We have rejected this evident option from the very beginning because in this case $\mu(z, m)$ will not be a meromorphic function of $z$ and in general one cannot write contour integrals for products of $\tilde{\Gamma}^{(r)}$-functions. However, following an early version of the present work, 
in [32] the rarefied elliptic hypergeometric functions are written using such a periodic gamma function. As a result, corresponding discrete parameters (as well as the discrete balancing condition) are automatically defined modulo $r$, which is not so in our case. The kernels of the corresponding sum-integrals become meromorphic functions of the integration variables $z_{j}$ only after imposing the balancing condition. As a result, in this case both normalizations of the gamma function appear to be equivalent.

\section{A RAREFiEd ELLiPtiC BETA INTEGRAL}

We define the kernel of a prospective rarefied elliptic beta integral

$$
\Delta_{\epsilon}^{(r)}\left(z, m ; t_{a}, n_{a} \mid p, q\right):=\frac{\prod_{a=1}^{6} \Gamma^{(r)}\left(t_{a} z, n_{a}+m+\epsilon ; p, q\right) \Gamma^{(r)}\left(t_{a} z^{-1}, n_{a}-m ; p, q\right)}{\Gamma^{(r)}\left(z^{ \pm 2}, \pm(2 m+\epsilon) ; p, q\right)},
$$

where we adopted the compact notation

$$
\Gamma^{(r)}\left(t z^{ \pm 1}, n \pm m ; p, q\right):=\Gamma^{(r)}(t z, n+m ; p, q) \Gamma^{(r)}\left(t z^{-1}, n-m ; p, q\right) .
$$

If the new discrete variable $\epsilon$ is an even integer, then the transformation

$$
n_{a} \rightarrow n_{a}-\epsilon / 2, \quad m \rightarrow m-\epsilon / 2
$$

removes $\epsilon$ completely. For odd $\epsilon$ using this transformation one can reduce the value of $\epsilon$ to 1 . Therefore we assume that $\epsilon$ takes the values

$$
\epsilon=0 \text { or } 1 \text {. }
$$

One has the permutation symmetry

$$
\Delta_{\epsilon}^{(r)}\left(z, m ; t_{a}, n_{a} \mid p, q\right)=\Delta_{-\epsilon}^{(r)}\left(z,-m ; t_{a},-n_{a} \mid q, p\right) .
$$

From now on, in most places we drop for brevity the bases $p$ and $q$ and the superscript $r$ from the notation for integrands and rarefied elliptic gamma functions.

It is not difficult to derive the following equations

$$
\frac{\Delta_{\epsilon}\left(p z, m-1 ; t_{a}, n_{a}\right)}{\Delta_{\epsilon}\left(z, m ; t_{a}, n_{a}\right)}=h_{1}(z, m), \quad \frac{\Delta_{\epsilon}\left(q z, m+1 ; t_{a}, n_{a}\right)}{\Delta_{\epsilon}\left(z, m ; t_{a}, n_{a}\right)}=h_{2}(z, m),
$$

where

$$
h_{1}(z, m)=\left(\frac{q^{2 m+\epsilon+1}}{p z^{2}}\right)^{\sum_{a=1}^{6} n_{a}+3 \epsilon} p q \prod_{a=1}^{6} \frac{\theta\left(t_{a} z q^{-n_{a}-m-\epsilon} ; q^{r}\right)}{\theta\left(t_{a}^{-1} p z q^{1+n_{a}-m} ; q^{r}\right)} \frac{\theta\left((p q z)^{2} q^{-2 m-\epsilon} ; q^{r}\right)}{\theta\left(z^{2} q^{-2 m-\epsilon} ; q^{r}\right)}
$$

and

$$
h_{2}(z, m)=\left(\frac{p^{2 m+\epsilon+1}}{q z^{2}}\right)^{\sum_{a=1}^{6} n_{a}+3 \epsilon} p q \prod_{a=1}^{6} \frac{\theta\left(t_{a} z p^{n_{a}+m+\epsilon} ; p^{r}\right)}{\theta\left(t_{a}^{-1} q z p^{1-n_{a}+m} ; p^{r}\right)} \frac{\theta\left((p q z)^{2} p^{2 m+\epsilon} ; p^{r}\right)}{\theta\left(z^{2} p^{2 m+\epsilon} ; p^{r}\right)} .
$$

One can check that

$$
\frac{h_{1}\left(q^{r} z, m\right)}{h_{1}(z, m)}=q^{2(1-r)\left(\sum_{a=1}^{6} n_{a}+3 \epsilon\right)} \frac{(p q)^{2}}{\prod_{a=1}^{6} t_{a}^{2}}, \quad \frac{h_{2}\left(p^{r} z, m\right)}{h_{2}(z, m)}=p^{2(r-1)\left(\sum_{a=1}^{6} n_{a}+3 \epsilon\right)} \frac{(p q)^{2}}{\prod_{a=1}^{6} t_{a}^{2}} .
$$


Therefore, imposing the balancing condition

$$
\prod_{a=1}^{6} t_{a}=p q, \quad \sum_{a=1}^{6} n_{a}+3 \epsilon=0
$$

we obtain

$$
h_{1}\left(q^{r} z, m\right)=h_{1}(z, m), \quad h_{2}\left(p^{r} z, m\right)=h_{2}(z, m) .
$$

Denoting $q=e^{2 \pi \mathrm{i} \sigma}, p=e^{2 \pi \mathrm{i} \tau}$, we see that $h_{1}\left(e^{2 \pi \mathrm{i} u}, m\right)$ becomes an elliptic function of $u$ of order 10 with the periods 1 and $r \sigma$, while $h_{2}\left(e^{2 \pi \mathrm{i} u}, m\right)$ becomes a similar function with the periods 1 and $r \tau$. Note that one could fix the balancing condition for the continuous parameters as $\prod_{a=1}^{6} t_{a}=-p q$, which also leads to elliptic functions, but, similar to the standard elliptic beta integral case [43, the choice (36) is a distinguished one.

One can verify that now $\Delta_{\epsilon}\left(t_{a}, n_{a} ; z, m\right)$ becomes a periodic function of $m$ :

$$
\Delta_{\epsilon}\left(z, m+r ; t_{a}, n_{a}\right)=\Delta_{\epsilon}\left(z, m ; t_{a}, n_{a}\right),
$$

since all quasiperiodicity factors emerging from the relation (25) cancel out. Using this fact we repeat $r$ times the recurrence relations (35) and obtain

$$
\begin{aligned}
& \Delta_{\epsilon}\left(p^{r} z, m ; t_{a}, n_{a}\right)=\prod_{k=0}^{r-1} h_{1}\left(p^{k} z, m-k\right) \Delta_{\epsilon}\left(z, m ; t_{a}, n_{a}\right), \\
& \Delta_{\epsilon}\left(q^{r} z, m ; t_{a}, n_{a}\right)=\prod_{k=0}^{r-1} h_{2}\left(q^{k} z, m+k\right) \Delta_{\epsilon}\left(z, m ; t_{a}, n_{a}\right) .
\end{aligned}
$$

Therefore, the function $\Delta_{\epsilon}\left(z, m ; t_{a}, n_{a}\right)$ is a solution of a finite-difference equation of the first order with the coefficient given by a particular elliptic function of order $10 r$.

We remind the reader of the definition of elliptic hypergeometric integrals in the multiplicative notation [44] - these are the contour integrals $\int_{C} \Delta(z) d z / z$ with $\Delta(z)$ satisfying the first order $q$-difference equation $\Delta(q z)=h(z) \Delta(z)$ with a $p$-periodic (i.e., elliptic) function $h(z), h(p z)=h(z)$. Therefore, if we consider a contour integral of our $\Delta_{\epsilon^{-}}$ function, by definition we obtain a standard elliptic hypergeometric integral with the bases $p$ and $q$ replaced by $p^{r}$ and $q^{r}$, respectively. If we further sum over $m$ we get an elliptic hypergeometric "sum-integral". We call such objects rarefied elliptic hypergeometric functions. Indeed, they are represented by sums of the standard elliptic hypergeometric integrals whose parameters are fixed in a particular way using the powers of $p^{1 / r}$ and $q^{1 / r}$ (in the notation of formula (9) ), which justifies the term "rarefied". Using the representation (31) one can rewrite the function $\Delta_{\epsilon}\left(z, m ; t_{a}, n_{a}\right)$ as a ratio of standard elliptic gamma functions with bases $p^{r}$ and $q^{r}$, however, the resulting expressions are cumbersome and we do not consider them here.

A rarefied analogue of the elliptic beta integral (9) has the following evaluation. 
Theorem 2. Let $t_{1}, \ldots, t_{6}, p, q \in \mathbb{C}^{*}$ and $n_{1}, \ldots, n_{6} \in \mathbb{Z}$ are such that $\left|t_{a}\right|,|p|,|q|<1$ and the following balancing condition holds true

$$
\prod_{a=1}^{6} t_{a}=p q, \quad \sum_{a=1}^{6} n_{a}+3 \epsilon=0, \quad \epsilon=0,1 .
$$

Then

$$
\kappa^{(r)} \sum_{m=0}^{r-1} \int_{\mathbb{T}} \Delta_{\epsilon}^{(r)}\left(z, m ; t_{a}, n_{a}\right) \frac{d z}{z}=\prod_{1 \leq a<b \leq 6} \Gamma^{(r)}\left(t_{a} t_{b}, n_{a}+n_{b}+\epsilon ; p, q\right),
$$

where $\kappa^{(r)}=\left(p^{r} ; p^{r}\right)_{\infty}\left(q^{r} ; q^{r}\right)_{\infty} / 4 \pi \mathrm{i}$ and $\mathbb{T}$ is the positively oriented unit circle.

For $\epsilon=0$ one gets the relation proven by Kels in [31]. The constraints $\left|t_{a}\right|<1$ can be relaxed by replacing for each fixed $m$ the integration contour $\mathbb{T}$ by a contour $C_{m}$ separating geometric progressions of the $\Delta_{\epsilon}$-function poles converging to zero from their reciprocals diverging to infinity. The conditions of existence of such contours are complicated, they impose certain constraints on the parameters and require thorough considerations. In this case, evidently, one cannot permute the summation over $m$ and integration over $z$ in (41). Note also that, because of the periodicity (38), the sum over $m=0,1, \ldots, r-1$ can be replaced by sums over any $r$ consecutive values of the integer $m$. Similarly, from the evident relation $\Delta_{\epsilon}\left(z,-m ; t_{a}, n_{a}\right)=\Delta_{\epsilon}\left(z^{-1}, m-\epsilon ; t_{a}, n_{a}\right)$ and the $r$-periodicity in $m$ one has

$$
\begin{aligned}
c_{r-m} & :=\int_{\mathbb{T}} \Delta_{\epsilon}\left(z, r-m ; t_{a}, n_{a}\right) \frac{d z}{z}=\int_{\mathbb{T}} \Delta_{\epsilon}\left(z^{-1}, m-\epsilon ; t_{a}, n_{a}\right) \frac{d z}{z} \\
& =\int_{\mathbb{T}} \Delta_{\epsilon}\left(z, m-\epsilon ; t_{a}, n_{a}\right) \frac{d z}{z}=c_{m-\epsilon} .
\end{aligned}
$$

Therefore the sum over $m$ in (41) can be reduced for $\epsilon=0$ to

$$
\sum_{m=0}^{r-1} c_{m}=\left\{\begin{array}{cl}
c_{0}+c_{r / 2}+2 \sum_{m=1}^{r / 2-1} c_{m} & \text { for even } r \\
c_{0}+2 \sum_{m=1}^{(r-1) / 2} c_{m} & \text { for odd } r
\end{array}\right.
$$

and for $\epsilon=1$ to

$$
\sum_{m=0}^{r-1} c_{m}=\left\{\begin{array}{cl}
2 \sum_{m=0}^{r / 2-1} c_{m} & \text { for even } r \\
c_{(r-1) / 2}+2 \sum_{m=0}^{(r-3) / 2} c_{m} & \text { for odd } r
\end{array}\right.
$$

We shall use such a representation in Sect. 6 for the consideration of a particular $p \rightarrow 0$ limit in the equality (41).

According to the discussion given above, on the left-hand side of identity (41) we have a sum of $r$ ordinary elliptic hypergeometric integrals. The proof of relation (41) different in certain aspects from the one used (for $\epsilon=0$ ) in [31] will be given in the next section as a subcase of a substantially more general situation. 


\section{A $C_{n}$ RAREFiEd ELLIPTIC BETA INTEGRAL OF TYPE I}

We define the kernel of the type I multiple rarefied elliptic beta integral for the root system $C_{n}$ as

$$
\begin{gathered}
\Delta_{I, \epsilon}\left(z_{j}, m_{j} ; t_{a}, n_{a}\right):=\prod_{1 \leq j<k \leq n} \frac{1}{\left.\Gamma\left(z_{j}^{ \pm 1} z_{k}^{ \pm 1}, \pm\left(m_{j}+\epsilon / 2\right) \pm\left(m_{k}+\epsilon / 2\right)\right)\right)} \\
\times \prod_{j=1}^{n} \frac{\prod_{a=1}^{2 n+4} \Gamma\left(t_{a} z_{j}, n_{a}+m_{j}+\epsilon\right) \Gamma\left(t_{a} z_{j}^{-1}, n_{a}-m_{j}\right)}{\Gamma\left(z_{j}^{ \pm 2}, \pm\left(2 m_{j}+\epsilon\right)\right)}
\end{gathered}
$$

where $t_{a}, z_{j} \in \mathbb{C}^{*}, n_{a}, m_{j} \in \mathbb{Z}, \epsilon=0,1$, and impose the balancing condition

$$
\prod_{a=1}^{2 n+4} t_{a}=p q, \quad \sum_{a=1}^{2 n+4} n_{a}+(n+2) \epsilon=0 .
$$

Association with the root system $C_{n}$ comes from the fact that the denominator of the ratio of the products of rarefied elliptic gamma functions in (44) can be formally written as $\prod_{\alpha \in R\left(C_{n}\right)} \Gamma\left(e^{u \alpha},(m+\epsilon / 2) \alpha ; p, q\right)$, where $\alpha \in\left\{ \pm e_{i} \pm e_{j}(i<j), \pm 2 e_{i}\right\}_{i, j=1, \ldots, n}$ are the roots of the root system $R\left(C_{n}\right)$ with $e_{i}$ being the standard euclidean basis vectors of $\mathbb{R}^{n}$ and $z_{i}:=e^{u e_{i}}, m_{i}:=m e_{i}$ for some formal variables $u \in \mathbb{C}$ and $m \in \mathbb{Z}$.

Theorem 3. Let $4 n+8$ continuous and discrete parameters $t_{a} \in \mathbb{C}^{*}, n_{a} \in \mathbb{Z}, a=$ $1, \ldots, 2 n+4$, the variable $\epsilon=0,1$, and bases $p, q \in \mathbb{C}$ satisfy the restrictions $|p|,|q|,\left|t_{a}\right|<1$ and the balancing condition (45). Denote

$$
\kappa_{n}^{(r)}=\frac{\left(p^{r} ; p^{r}\right)_{\infty}^{n}\left(q^{r} ; q^{r}\right)_{\infty}^{n}}{(4 \pi \mathrm{i})^{n} n !}
$$

Then

$$
\kappa_{n}^{(r)} \sum_{m_{1}, \ldots, m_{n}=0}^{r-1} \int_{\mathbb{T}^{n}} \Delta_{I, \epsilon}\left(z_{j}, m_{j} ; t_{a}, n_{a}\right) \prod_{j=1}^{n} \frac{d z_{j}}{z_{j}}=\prod_{1 \leq a<b \leq 2 n+4} \Gamma\left(t_{a} t_{b}, n_{a}+n_{b}+\epsilon ; p, q\right),
$$

where $\mathbb{T}$ is the unit circle of positive orientation.

Proof. For proving this identity we adapt to the present situation the proof of the standard type I $C_{n}$-integral evaluation formula given in [47]. As we will see, several key steps will be identical. First, let us remove parameters $t_{2 n+4}$ and $n_{2 n+4}$ using the balancing constraint. For that we denote

$$
A:=\prod_{a=1}^{2 n+3} t_{a}=\frac{p q}{t_{2 n+4}}, \quad N:=\sum_{a=1}^{2 n+3} n_{a}+(n+2) \epsilon=-n_{2 n+4},
$$

and apply the inversion relation for the rarefied elliptic gamma function involving parameter $t_{2 n+4}$. Now we divide the left-hand side of equality (46) by $\kappa_{n}^{(r)}$ times the expression 
on the right-hand side and rewrite it as

$$
I_{\epsilon}\left(t_{1}, \ldots, t_{2 n+3}, n_{1}, \ldots, n_{2 n+3}\right):=\sum_{m=0}^{r-1} \int_{\mathbb{T}} \rho_{\epsilon}\left(z_{j}, m_{j} ; t_{a}, n_{a}\right) \prod_{j=1}^{n} \frac{d z_{j}}{z_{j}}=\frac{1}{\kappa_{n}^{(r)}},
$$

where

$$
\begin{aligned}
\rho_{\epsilon}\left(z_{j}, m_{j} ; t_{a}, n_{a}\right) & :=\prod_{1 \leq j<k \leq n} \frac{1}{\Gamma\left(z_{j}^{ \pm 1} z_{k}^{ \pm 1}, \pm\left(m_{j}+\epsilon / 2\right) \pm\left(m_{k}+\epsilon / 2\right)\right)} \\
\times & \prod_{j=1}^{n} \frac{\prod_{a=1}^{2 n+3} \Gamma\left(t_{a} z_{j}, n_{a}+m_{j}+\epsilon\right) \Gamma\left(t_{a} z_{j}^{-1}, n_{a}-m_{j}\right)}{\Gamma\left(z_{j}^{ \pm 2}, \pm\left(2 m_{j}+\epsilon\right)\right) \Gamma\left(A z_{j}^{-1}, N-m_{j}-\epsilon\right) \Gamma\left(A z_{j}, N+m_{j}\right)} \\
& \times \frac{\prod_{a=1}^{2 n+3} \Gamma\left(A t_{a}^{-1}, N-n_{a}-\epsilon\right)}{\prod_{1 \leq a<b \leq 2 n+3} \Gamma\left(t_{a} t_{b}, n_{a}+n_{b}+\epsilon\right)} .
\end{aligned}
$$

This $\rho_{\epsilon}$-function is $r$-periodic in all discrete variables $m_{j}, j=1, \ldots, n$, and $n_{a}, a=$ $1, \ldots, 2 n+3$

$$
\rho_{\epsilon}\left(z_{j}, \ldots, m_{k}+r, \ldots\right)=\rho_{\epsilon}\left(z_{j}, \ldots, n_{b}+r, \ldots\right)=\rho_{\epsilon}\left(z_{j}, m_{j} ; t_{a}, n_{a}\right),
$$

which is a very important property following from a lengthy cancellation of the complicated quasiperiodicity multipliers generated by the rarefied elliptic gamma functions.

Let us investigate the divisor of (48) considered as a function of $z_{\ell}$. Due to the property

$$
\Gamma^{(r)}(z, m) \Gamma^{(r)}\left(z^{-1},-m\right)=\frac{(p q)^{\frac{m(m+1)}{2}}}{\theta\left(z q^{-m} ; q^{r}\right) \theta\left(z^{-1} p^{-m} ; p^{r}\right)},
$$

it does not contain poles whose positions do not depend on $t_{a}$ (at $z_{\ell}=0$ one has an essential singularity). The $t_{a}$-independent zeros do not play any role in the following considerations and we skip them. As to the $t_{a}$-dependent poles and zeros, generically, the function $\rho_{\epsilon}\left(z_{\ell}, \ldots\right)$ has sequences of poles converging to $z_{\ell}=0$ for any $\ell$ by the points of the sets

$$
P_{\text {in }}^{A}=\left\{t_{a} q^{k} p^{n_{a}-m_{\ell}+k+r j}\right\}, \quad P_{\text {in }}^{B}=\left\{t_{a} q^{r-n_{a}+m_{\ell}+k+r j} p^{k}\right\}
$$

with $a=1, \ldots, 2 n+4$ and $j, k \in \mathbb{Z}_{\geq 0}$, and going to infinity along the sets

$$
P_{\text {out }}^{A}=\left\{t_{a}^{-1} q^{-k} p^{-n_{a}-m_{\ell}-\epsilon-k-r j}\right\}, \quad P_{\text {out }}^{B}=\left\{t_{a}^{-1} q^{n_{a}+m_{\ell}+\epsilon-k-r(j+1)} p^{-k}\right\},
$$

which are not identically $z \rightarrow 1 / z$ reciprocal to $P_{\text {in }}$. Zeros of this function converge to $z_{\ell}=0$ for any $\ell$ by the point sets

$$
Z_{\mathrm{in}}^{A}=\left\{t_{a}^{-1} q^{k+1} p^{-n_{a}-m_{\ell}-\epsilon+k+1+r(j+1)}\right\}, \quad Z_{\mathrm{in}}^{B}=\left\{t_{a}^{-1} q^{n_{a}+m_{\ell}+\epsilon+k+1+r j} p^{k+1}\right\}
$$

with $a=1, \ldots, 2 n+4$ and $j, k \in \mathbb{Z}_{\geq 0}$, and go to infinity along the point sets

$$
Z_{\text {out }}^{A}=\left\{t_{a} q^{-k-1} p^{n_{a}-m_{\ell}-k-1-r(j+1)}\right\}, \quad Z_{\text {out }}^{B}=\left\{t_{a} q^{-n_{a}+m_{\ell}-k-1-r j} p^{-k-1}\right\},
$$

which are also not identically $z \rightarrow 1 / z$ reciprocal to $Z_{\text {in }}$.

As one can see, the structure of poles and zeros is rather complicated and it may happen that in the sets indicated above positions of some poles and zeros coincide and, actually, both are absent. First, we assume that the parameters $t_{a}$ and bases $p, q$ are 
multiplicatively incommensurate, i.e. $t_{a}^{n} t_{b}^{m} p^{k} q^{l} \neq 1$ for $n, m, k, l \in \mathbb{Z}$, which guarantees that all poles and zeros are simple. Then, equating positions of poles and zeros (with $j, k$ replaced by $j^{\prime}, k^{\prime}$ ), we find that nontrivial cancellations may exist only if $j=j^{\prime}$ and either

$$
r j+k+k^{\prime}=-n_{a}+m_{\ell}-1
$$

if there are intersecting points in $P_{\text {in }}^{A}$ and $Z_{\text {out }}^{B}$, or

$$
r j+k+k^{\prime}=n_{a}-m_{\ell}-r-1,
$$

if $P_{\text {in }}^{B}$ and $Z_{\text {out }}^{A}$ overlap, or

$$
r j+k+k^{\prime}=-n_{a}-m_{\ell}-\epsilon-1,
$$

if $P_{\text {out }}^{A}$ and $Z_{\text {in }}^{B}$ overlap, or

$$
r j+k+k^{\prime}=n_{a}+m_{\ell}+\epsilon-r-1,
$$

if $P_{\text {out }}^{B}$ and of $Z_{\text {in }}^{A}$ overlap. Let us denote as $p_{\text {in }}^{\max }$ the maximal possible absolute value of the pole positions in some indicated subset of $P_{\text {in }}$ and as $p_{\text {out }}^{\text {min }}$ the minimal possible absolute value of the pole positions in some indicated subset of $P_{\text {out }}$.

Recall that $j, k, k^{\prime} \geq 0$, but $n_{a}$ and $m_{\ell}$ can take arbitrary integer values. The periodicity (49) means that the poles of the $\rho_{\epsilon}$-function form a periodic lattice in $n_{a}$ and $m_{\ell}$ and the above equations for $j, k, k^{\prime}$ always have solutions for sufficiently large $\left|n_{a}\right|$ and $|m|$, in which case a part of the poles is cancelled by zeros. Therefore, without loss of generality, we can restrict the values of $m$ and $n_{a}$ to

$$
0 \leq m \leq r-1, \quad-r<n_{a}<r
$$

(this can be done simply by the shifts $n_{a} \rightarrow n_{a} \pm r$ and $n_{2 n+4} \rightarrow n_{2 n+4} \mp r$, as soon as one gets $\left|\sum_{k=1}^{a}\left(n_{k}+\epsilon / 2\right)\right| \geq r$ for $a=1, \ldots, 2 n+3$. As a result, we have $-2 r+1<n_{a}-m_{\ell}<r$ for all $a$, which means that equation (51) has no solutions and $p_{\text {in, }, B}^{\max }=\max \left|q t_{a}\right|$. Here and until formula (54) below we assume that max and min values are taken in the parameter sets with the index $a=1, \ldots, 2 n+4$.

Suppose now that $n_{a}-m_{\ell} \geq 0$. Then equation (50) has no solutions and $p_{\mathrm{in}, A}^{\max }=$ $\max \left|t_{a}\right|$, which is reached only for $m_{\ell}=n_{a}$ with the corresponding value of $a$. Let now $m_{\ell} \geq n_{a}+1$. Then equation (50) may have nontrivial solutions. For $j=0$ one has $k, k^{\prime}=0,1, \ldots, m_{\ell}-n_{a}-1$, so that $p_{\mathrm{in}, A, j=0}^{\max }=\max \left|q t_{a}\right|$. For $j=1$ and $0<m_{\ell}-n_{a}<r+1$ there are no solutions and the maximal absolute value of the corresponding pole positions is $\max \left|t_{a}\right|$. For $j=1$ and $m_{\ell}-n_{a} \geq r+1$ (which can be satisfied only for $r>2$ ) the solution is $k, k^{\prime}=0,1, \ldots, m_{\ell}-n_{a}-1-r$, and the top possible pole position has the absolute value $\max \left|q t_{a}\right|$. So, $p_{\mathrm{in}, A, j=1}^{\max }=\max \left|t_{a}\right|$. The poles in $P_{\mathrm{in}}^{A}$ with $j>1$ have $p_{\text {in }, A, j>1}^{\max }=\max \left|p^{2} t_{a}\right|$. So, for $\left|t_{a}\right|<1$ all the poles from $P_{\text {in }}$ lie inside $\mathbb{T}$.

Similar situation takes place for the poles $P_{\text {out }}$ and zeros $Z_{\text {in }}$. Indeed, for $n_{a}+m_{\ell}+\epsilon \geq 0$ there are no solutions of equation (52) with $p_{\mathrm{out}, A}^{\min }=\min \left|t_{a}^{-1}\right|$, which is reached for $m_{\ell}=$ $-n_{a}-\epsilon$ with the corresponding value of $a$. For $m_{\ell}<-n_{a}-\epsilon$ the solution of (152) is $j=0$ and $k, k^{\prime}=0, \ldots,-n_{a}-m_{\ell}-\epsilon-1$ with $p_{\text {out }, A, j=0}^{\min }=\min \left|t_{a}^{-1} q^{-1}\right|$. The poles with $j>0$ have $p_{\text {out }, A, j>0}^{\min }=\min \left|t_{a}^{-1} p^{-1}\right|($ for $\epsilon=0)$. 
Consider now $P_{\text {out }}^{B}$. For $n_{a}+m_{\ell}+\epsilon \leq r$ equation (153) has no solutions and $p_{\text {out }, B}^{\min }=$ $\min \left|t_{a}^{-1}\right|$, which is reached for $m_{\ell}=r-n_{a}-\epsilon$ with the corresponding value of $a$. For $n_{a}+m_{\ell}+\epsilon>r$ (which can be satisfied only for $r>2$ ) the solutions of (53) have the form $j=0$ and $k, k^{\prime}=0, \ldots, n_{a}+m_{\ell}+\epsilon-r-1$ with $p_{\mathrm{out}, B, j=0}^{\min }=\min \left|t_{a}^{-1} p^{-1}\right|$. Finally, for $j>0$ equation (53) has no solutions and one has $p_{\text {out }, B, j>0}^{\min }=\min \left|t_{a}^{-1} q^{-1}\right|($ for $\epsilon=1)$. So, all the poles from $P_{\text {out }}$ lie outside $\mathbb{T}$ for $\left|t_{a}\right|<1$.

To conclude, if we impose the constraint $\max \left|t_{a}\right|<1$, then all poles from $P_{\text {in }}$ and $P_{\text {out }}$ are pushed inside and outside of $\mathbb{T}$, respectively. It is exactly this property (which is established after a rather neat analysis of the structure of $\rho_{\epsilon}$-function divisor points) that determines the choice of $\mathbb{T}$ as the integration contour in formula (46).

Now we prove the following finite-difference equation:

$$
\begin{aligned}
\rho_{\epsilon}\left(z_{j}, m_{j} ; p\right. & \left.t_{1}, t_{2}, \ldots, n_{1}-1, n_{2}, \ldots\right)-\rho_{\epsilon}\left(z_{j}, m_{j} ; t_{a}, n_{a}\right) \\
& =\sum_{k=1}^{n}\left(g_{k, \epsilon}\left(\ldots, p^{-1} z_{k}, \ldots, m_{k}+1, \ldots ; t_{a}, n_{a}\right)-g_{k, \epsilon}\left(z_{j}, m_{j} ; t_{a}, n_{a}\right)\right),
\end{aligned}
$$

where

$$
\begin{gathered}
g_{k, \epsilon}\left(z_{j}, m_{j} ; t_{a}, n_{a}\right)=\rho_{\epsilon}\left(z_{j}, m_{j} ; t_{a}, n_{a}\right) \prod_{\substack{\ell=1 \\
\ell \neq k}}^{n} \frac{\theta\left(t_{1} z_{\ell} q^{-n_{1}-m_{\ell}-\epsilon}, t_{1} z_{\ell}^{-1} q^{-n_{1}+m_{\ell}} ; q^{r}\right)}{\theta\left(z_{k} z_{\ell} q^{-m_{k}-m_{\ell}-\epsilon}, z_{k} z_{\ell}^{-1} q^{-m_{k}+m_{\ell}} ; q^{r}\right)} \\
\times \frac{\prod_{a=1}^{2 n+3} \theta\left(t_{a} z_{k} q^{-n_{a}-m_{k}-\epsilon} ; q^{r}\right)}{\prod_{a=2}^{2 n+3} \theta\left(t_{1} t_{a} q^{-n_{1}-n_{a}-\epsilon} ; q^{r}\right)} \frac{\theta\left(t_{1} A q^{-n_{1}-N} ; q^{r}\right)}{\theta\left(z_{k}^{2} q^{-2 m_{k}-\epsilon}, A z_{k} q^{-N-m_{k}} ; q^{r}\right)} \frac{t_{1} q^{m_{k}}}{z_{k} q^{n_{1}}} .
\end{gathered}
$$

Dividing this equation by $\rho_{\epsilon}\left(z_{j}, m_{j} ; t_{a}, n_{a}\right)$ we come to the following identity

$$
\begin{aligned}
& \prod_{j=1}^{n} \frac{\theta\left(t_{1} z_{j} q^{-n_{1}-m_{j}-\epsilon}, t_{1} z_{j}^{-1} q^{-n_{1}+m_{j}} ; q^{r}\right)}{\theta\left(A z_{j} q^{-N-m_{j}}, A z_{j}^{-1} q^{-N+m_{j}+\epsilon} ; q^{r}\right)} \prod_{a=2}^{2 n+3} \frac{\theta\left(A t_{a}^{-1} q^{-N+n_{a}+\epsilon} ; q^{r}\right)}{\theta\left(t_{1} t_{a} q^{-n_{1}-n_{a}-\epsilon} ; q^{r}\right)}-1 \\
& =\frac{t_{1} q^{-n_{1}} \theta\left(t_{1} A q^{-n_{1}-N} ; q^{r}\right)}{\prod_{a=2}^{2 n+3} \theta\left(t_{1} t_{a} q^{-n_{1}-n_{a}-\epsilon} ; q^{r}\right)} \sum_{k=1}^{n} \frac{q^{m_{k}}}{z_{k} \theta\left(z_{k}^{2} q^{-2 m_{k}-\epsilon} ; q^{r}\right)} \\
& \times \prod_{\substack{j=1 \\
j \neq k}}^{n} \frac{\theta\left(t_{1} z_{j} q^{-n_{1}-m_{j}-\epsilon}, t_{1} z_{j}^{-1} q^{-n_{1}+m_{j}} ; q^{r}\right)}{\theta\left(z_{k} z_{j} q^{-m_{k}-m_{j}-\epsilon}, z_{k} z_{j}^{-1} q^{-m_{k}+m_{j}} ; q^{r}\right)} \\
& \times\left(\frac{z_{k}^{2 n+2} \prod_{a=1}^{2 n+3} \theta\left(t_{a} z_{k}^{-1} q^{-n_{a}+m_{k}} ; q^{r}\right)}{q^{(n+1)\left(2 m_{k}+\epsilon\right)} \theta\left(A z_{k}^{-1} q^{-N+m_{k}+\epsilon} ; q^{r}\right)}-\frac{\prod_{a=1}^{2 n+3} \theta\left(t_{a} z_{k} q^{-n_{a}-m_{k}-\epsilon} ; q^{r}\right)}{\theta\left(A z_{k} q^{-N-m_{k}} ; q^{r}\right)}\right) .
\end{aligned}
$$

The shifts $z_{j} \rightarrow z_{j} q^{m_{j}+\epsilon / 2}$ and $t_{a} \rightarrow t_{a} q^{n_{a}+\epsilon / 2}$ remove completely the discrete variables $m_{j}$ $n_{a}$ and $\epsilon$ from (56) and we obtain precisely the elliptic functions identity established in [47] in the proof of the type I $C_{n}$-integral (with $p$ and $q$ replaced by $p^{r}$ and $q^{r}$ ).

We now integrate equation (54) over the multi-contour $\mathbb{T}^{n}$ and sum over all $m_{j}$ from 0 to $r-1$. It can be checked that $g_{k, \epsilon}$-functions are periodic with respect to the shifts 
$m_{j} \rightarrow m_{j}+r$. Therefore we obtain

$$
\begin{aligned}
& I_{\epsilon}\left(p t_{1}, t_{2}, \ldots, t_{2 n+3}, n_{1}-1, n_{2}, \ldots, n_{2 n+3}\right)-I_{\epsilon}\left(t_{a}, n_{a}\right) \\
& \quad=\sum_{m_{1}, \ldots, m_{n}=0}^{r-1} \sum_{\ell=1}^{n}\left(\int_{\mathbb{T}^{\ell-1} \times\left(p^{-1} \mathbb{T}\right) \times \mathbb{T}^{n-\ell}}-\int_{\mathbb{T}^{n}}\right) g_{\ell, \epsilon}\left(z_{j}, m_{j} ; t_{a}, n_{a}\right) \prod_{j=1}^{n} \frac{d z_{j}}{z_{j}},
\end{aligned}
$$

where $p^{-1} \mathbb{T}$ denotes the contour obtained from $\mathbb{T}$ after scaling it by $p^{-1}$.

The divisor points of the functions $g_{\ell}$ (55) in the variable $z_{\ell}$ are determined by the following factor (theta functions were absorbed into the gamma functions by appropriate shifts of the arguments):

$$
\begin{aligned}
& \prod_{a=1}^{2 n+3}\left[\Gamma\left(p t_{a} z_{\ell}, n_{a}+m_{\ell}+\epsilon-1\right) \Gamma\left(t_{a} z_{\ell}^{-1}, n_{a}-m_{\ell}\right)\right] \\
& \quad \times \Gamma\left(t_{2 n+4} z_{\ell}, n_{2 n+4}+m_{\ell}+\epsilon\right) \Gamma\left(p^{-1} t_{2 n+4} z_{\ell}^{-1}, n_{2 n+4}-m_{\ell}+1\right) .
\end{aligned}
$$

Comparing with the previous analysis of the divisor of the $\rho_{\epsilon}$-function, we see that the equations (50) and (51) are preserved for $P_{\text {in }}$ poles associated with $t_{a}, a=1, \ldots, 2 n+3$, whereas, vice versa, equations (52) and (53) remain the same for $P_{\text {out }}$ poles associated with $t_{2 n+4}$. The sum $r j+k+k^{\prime}$ is equal to $-n_{2 n+4}+m_{\ell}-2$ or $n_{2 n+4}-m_{\ell}-r$ for the analogues of (50) and (51) with $a=2 n+4$, respectively, and to $-n_{a}-m_{\ell}-\epsilon$ or $n_{a}+m_{\ell}+\epsilon-r-2$ for the analogues of (52) and (53) with $a=1, \ldots, 2 n+3$. As a result of such changes we find that $p_{\text {in }}^{\max }=\max \left\{\left|t_{a}\right|,\left|p^{-1} t_{2 n+4}\right|\right\}$ and $p_{\text {out }}^{\min }=\min \left\{\left|t_{2 n+4}^{-1}\right|,\left|p^{-1} t_{a}\right|\right\}$, where $a=1, \ldots, 2 n+3$.

Therefore, for $\left|t_{a}\right|<1, a=1, \ldots, 2 n+3$, and $\left|t_{2 n+4}\right|<|p|$ the functions $g_{\ell}$ do not have poles in the annuli $1 \leq\left|z_{\ell}\right| \leq\left|p^{-1}\right|$. As a result, we can safely shrink the integration contour $p^{-1} \mathbb{T}$ to $\mathbb{T}$ in (57) and obtain zero on the right-hand side, i.e. the equality

$$
I_{\epsilon}\left(p t_{1}, t_{1}, \ldots, n_{1}-1, n_{2}, \ldots\right)=I_{\epsilon}\left(t_{a}, n_{a}\right) .
$$

Note that for the taken constraints on the parameters the contour $\mathbb{T}$ is legitimate for both integrals on the left-hand side of (57), i.e. it separates relevant sets of poles.

Due to the incommensurability condition, the integral $I_{\epsilon}\left(t_{a}, n_{a}\right)$ is a meromorphic function of the parameters $t_{a}$. Therefore, equation (58) can be used for analytic continuation of $I_{\epsilon}\left(t_{a}, n_{a}\right)$ from the domain $\left|t_{1}\right|,\left|t_{2 n+4}\right|<1$ to $\left|p^{k} t_{1}\right|,\left|p^{-k} t_{2 n+4}\right|<1$ for any $k \in \mathbb{Z}$. Therefore, iterating (58) $r$ times and using the periodicity property

$$
I_{\epsilon}\left(\ldots, n_{b-1}, n_{b}+r, n_{b+1}, \ldots\right)=I_{\epsilon}\left(t_{a}, n_{a}\right), \quad b=1, \ldots, 2 n+3,
$$

following from the $\rho_{\epsilon}$-function periodicity in variables $n_{b}$, we obtain the equality

$$
I_{\epsilon}\left(p^{r} t_{1}, t_{2}, \ldots, n_{a}\right)=I_{\epsilon}\left(t_{a}, n_{a}\right) .
$$

Let us impose the additional constraint $\left|t_{2 n+4}\right|<|q|$. Then we can permute bases $p$ and $q$ in the above considerations, apply the symmetry (26) and obtain the equality

$$
I_{\epsilon}\left(q^{r} t_{1}, t_{2}, \ldots,-\epsilon-n_{a}\right)=I_{\epsilon}\left(t_{a},-\epsilon-n_{a}\right) .
$$


However, the integral $I_{\epsilon}$ is $r$-periodic in $n_{a}$-variables, i.e. the signs of $n_{a}$ do not matter. Therefore, we have

$$
I_{\epsilon}\left(p^{r} t_{1}, t_{2}, \ldots, n_{a}\right)=I_{\epsilon}\left(q^{r} t_{1}, t_{2}, \ldots, n_{a}\right)=I_{\epsilon}\left(t_{1}, t_{2}, \ldots, n_{a}\right)
$$

Since our bases $p$ and $q$ are incommensurate, this means that $I_{\epsilon}\left(t_{a}, n_{a}\right)$ does not depend on the parameter $t_{1}$ and, by symmetry, on all parameters $t_{a}$. Substituting this condition into recursion (58), we find that, actually, $I_{\epsilon}\left(t_{a}, n_{a}\right)$ does not depend on $n_{a}$ as well, i.e. it is a constant depending only on $p, q, r, n$ and $\epsilon, I_{\epsilon}\left(t_{a}, n_{a}\right)=c_{\epsilon}(p, q, r, n)$. Let us compute this constant $c_{\epsilon}$.

For that we set

$$
n_{a}=0, a=1, \ldots, n+2, \quad n_{a}=-\epsilon, a=n+3, \ldots, 2 n+4,
$$

which satisfies the discrete balancing condition, and consider the limit

$$
t_{a} t_{a+n+2} \rightarrow 1, \quad a=1, \ldots, n
$$

Our analysis of the $\rho_{\epsilon}$-function divisor structure shows that in each summation over the discrete variables $0 \leq m_{j} \leq r-1$ there is one integral, corresponding to the value $m_{j}=0$, for which the integration contour $\mathbb{T}$ becomes pinched by $2 n$ pairs of poles. The $\rho_{\epsilon}$-function contains the factor $1 / \prod_{j=1}^{n} \Gamma\left(t_{j} t_{j+n+2}, 0\right)$ which vanishes unless it is cancelled by the residues of poles pinching the integration contour for all $n$ integrals simultaneously. Therefore, our problem reduces to computation of the limit

$$
\begin{aligned}
& \lim _{\substack{t_{a} t_{a+n+2} \rightarrow 1 \\
a=1, \ldots, n}} \mu_{\epsilon}\left(t_{a}\right) \int_{\mathbb{T}^{n}} \prod_{1 \leq j<k \leq n} \frac{1}{\Gamma\left(\left(z_{j} z_{k}\right)^{ \pm 1}, \pm \epsilon\right) \Gamma\left(\left(z_{j} / z_{k}\right)^{ \pm 1}, 0\right)} \\
& \quad \times \prod_{j=1}^{n} \frac{\prod_{a=1}^{n+2} \Gamma\left(t_{a} z_{j}, \epsilon\right) \Gamma\left(t_{a+n+2} z_{j}, 0\right) \Gamma\left(t_{a} z_{j}^{-1}, 0\right) \Gamma\left(t_{a+n+2} z_{j}^{-1},-\epsilon\right)}{\Gamma\left(z_{j}^{ \pm 2}, \pm \epsilon\right)} \frac{d z_{j}}{z_{j}}
\end{aligned}
$$

where

$$
\frac{1}{\mu_{\epsilon}\left(t_{a}\right)}:=\prod_{1 \leq a<b \leq n+2} \Gamma\left(t_{a} t_{b}, \epsilon\right) \Gamma\left(t_{a+n+2} t_{b+n+2},-\epsilon\right) \prod_{1 \leq a, b \leq n+2} \Gamma\left(t_{a} t_{b+n+2}, 0\right) .
$$

Before taking the limits $t_{a} t_{a+n+2} \rightarrow 1, a=1, \ldots, n$, we deform each $\mathbb{T}$ to a contour $C$ which crosses the poles $z_{j}=t_{a}, t_{a+n+2}, a=1, \ldots, n$, and does not touch other poles. Again, the result does not vanish for $t_{a} t_{a+n+2}=1$ only if we pick up residues for all variables $z_{j}$ simultaneously. Whenever two different variables $z_{j}$ pick up residues from identical pole positions, we get zero due to the functions $\Gamma\left(\left(z_{j} / z_{k}\right)^{ \pm 1}, 0\right)$ in the integrand's denominator. Therefore, we should consider only the residues for $z_{j}=t_{j}$ and their $n$ ! permutations giving identical results. The residues for $z_{j}=t_{j+n+2}$ give the same result, 
which results in the additional multiplier $2^{n}$. So, the limit of interest is equal to

$$
\begin{gathered}
n !(4 \pi \mathrm{i})^{n} \lim _{\substack{t_{a} t_{a+n+2 \rightarrow 1} \\
a=1, \ldots, n}} \mu_{\epsilon}\left(t_{a}\right) \frac{\prod_{j=1}^{n} \prod_{a=1}^{n+2} \Gamma\left(t_{a} t_{j}, \epsilon\right) \Gamma\left(t_{a+n+2} t_{j}, 0\right) \Gamma\left(t_{a+n+2} t_{j}^{-1},-\epsilon\right)}{\prod_{1 \leq j<k \leq n} \Gamma\left(\left(t_{j} t_{k}\right)^{ \pm 1}, \pm \epsilon\right) \Gamma\left(\left(t_{j} / t_{k}\right)^{ \pm 1}, 0\right) \prod_{j=1}^{n} \Gamma\left(t_{j}^{ \pm 2}, \pm \epsilon\right)} \\
\times \prod_{j=1}^{n} \prod_{a=1}^{n+2} \lim _{z_{j} \rightarrow t_{j}}\left(1-t_{j} z_{j}^{-1}\right) \Gamma\left(t_{a} z_{j}^{-1}, 0\right) .
\end{gathered}
$$

We apply now the limiting relation (29) and cancel common factors from the numerator and denominator. The remaining gamma functions disappear after substitution of the relations $t_{a} t_{a+n+2}=1, a=1, \ldots, n+2$, and $t_{n+1} t_{n+2} t_{2 n+3} t_{2 n+4}=p q$ due to the inversion relation (27). As a result,

$$
c_{\epsilon}(p, q, r, n)=\left.\lim _{\substack{t_{a} t_{a+n+2 \rightarrow 1} \\ a=1, \ldots, n}} I_{\epsilon}\left(t_{j}, n_{j}\right)\right|_{\substack{n_{j}=n_{j+n+2}+\epsilon=0 \\ j=1, \ldots, n+2}}=\frac{(4 \pi \mathrm{i})^{n} n !}{\left(p^{r} ; p^{r}\right)_{\infty}^{n}\left(q^{r} ; q^{r}\right)_{\infty}^{n}}=\frac{1}{\kappa_{n}^{(r)}},
$$

as required.

Finally, by analytic continuation, we relax the restrictions $\left|t_{2 n+4}\right|<|p|,|q|$ to $\left|t_{2 n+4}\right|<1$ and remove the incommensurability constraint $t_{a}^{n} t_{b}^{m} p^{k} q^{l} \neq 1, n, m, k, l \in \mathbb{Z}$ (still keeping $\left.\left|t_{a}\right|,|p|,|q|<1\right)$. The theorem is proved.

Evidently, in the final result (46) one can relax restrictions for the $t_{a}$-parameters values by changing $\mathbb{T}$ to a contour $C$ such that it separates the poles $P_{\text {in }}$ and $P_{\text {out }}$ for all possible values of $m_{\ell}$. However, the analysis of sufficiency conditions for existence of such a contour is a complicated task and we do not consider it here.

\section{A special $p \rightarrow 0$ LIMIT}

Let us rewrite $n=1$ relation (41) in terms of the $\gamma^{(r)}$-functions. The left-hand side expression takes the form

$$
\prod_{a=1}^{6}\left[\frac{t_{a}^{\left(n_{a}+\epsilon / 2\right)\left(n_{a}+\epsilon / 2-1\right)}}{p^{\left(n_{a}+\epsilon / 2\right)^{2}}}\left(\frac{p}{q}\right)^{\frac{1}{3}\left(n_{a}+\epsilon / 2\right)^{3}}\right] \sum_{m=0}^{r-1} c_{m}\left(n_{a}, \epsilon\right),
$$

where

$$
c_{m}\left(n_{a}, \epsilon\right)=\kappa^{(r)}\left(\frac{q}{p}\right)^{(m+\epsilon / 2)^{2}} \int_{\mathbb{T}} \frac{\prod_{a=1}^{6} \gamma^{(r)}\left(t_{a} z, n_{a}+m+\epsilon\right) \gamma^{(r)}\left(t_{a} z^{-1}, n_{a}-m\right)}{z^{2 m+\epsilon} \gamma^{(r)}\left(z^{ \pm 2}, \pm(2 m+\epsilon)\right)} \frac{d z}{z}
$$

(note that the integrand is an explicit meromorphic function of $z \in \mathbb{C}^{*}$ ). The right-hand side can be "simplified" in a similar way and, after cancelling common factors, we obtain the relation

$$
\sum_{m=0}^{r-1} c_{m}\left(n_{a}, \epsilon\right)=(-1)^{\epsilon} \prod_{a=1}^{6} \frac{(q / p)^{\frac{1}{2}\left(n_{a}+\epsilon / 2\right)^{2}}}{t_{a}^{n_{a}+\epsilon / 2}} \prod_{1 \leq a<b \leq 6} \gamma^{(r)}\left(t_{a} t_{b}, n_{a}+n_{b}+\epsilon\right) .
$$

Now we can compare our proof of this relation for $\epsilon=0$ with the one suggested by Kels in [31]. In [31] the function $\llbracket m \rrbracket:=m \bmod r$ is used which is not needed in our 
consideration, since all necessary finite-dimensional truncations are guaranteed by the $r$-periodicity in all discrete variables of the integrand in (47). This makes our formulae uniform in the values of discrete variables $n_{a}$ and $m$ in contrast with [31], where they were brought to the values $1, \ldots, r-1$ by subtracting or adding multiples of $r$.

The kernel of sum-integral of [31] has the same $\gamma^{(r)}$-dependent part as above (with $\epsilon=0$ ), but the multiplier in front of it looks substantially different. In particular, for $n_{a}=0$ it misses our $z^{-2 m}$ factor. However, discrete arguments of the corresponding gamma functions involve $\llbracket \ell$, for some integers $\ell$, instead of simple $\ell$ in our case. It appears, that after replacing $\llbracket \ell \rrbracket$ by $\ell+k r$ for appropriate values of $k$ bringing $\ell+k r$ to the values $0,1, \ldots, r-1$ and applying the quasiperiodicity relations (21), our identity (41) coincides in this case with the one suggested in [31].

Since we use different normalizing factor for the rarefied elliptic gamma function (24), even after clearing the arguments of gamma functions from $\llbracket \ell \rrbracket$-functions, the left-hand and right-hand sides of our identities do not coincide (but the ratio does). Next, an analogue of the relation (56) in [31] contains formal fractional powers of $z$ and it looks

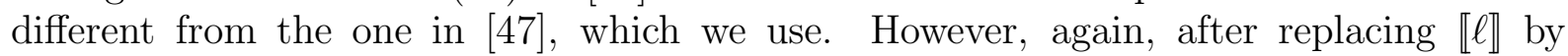
appropriate expressions $\ell+k r$, these fractional powers disappear and the finite-difference equation used in [31] becomes identical with ours. So, the approach suggested in this work is equivalent to the one in 31, but, to the author's taste, it looks more natural. Another point is that the analysis of the divisor points of the integrands in our case is essentially more detailed, since we check case by case the possibilities of cancellation of poles and zeros, whereas is [31] the divisor was forced to take a particular form by the use of $\llbracket \ell \rrbracket$-functions.

Inspired by the first version of this paper, the authors of [23] suggested to use the following periodic gamma function

$$
\begin{gathered}
\tilde{\Gamma}^{(r)}(z, m ; p, q):=\left(\frac{z}{\sqrt{p q}}\right)^{\frac{m(m-r)}{2 r}}\left(e^{-\pi \mathrm{i}} \sqrt{\frac{p}{q}}\right)^{\frac{m(m-r)(2 m-r)}{6 r}} \gamma^{(r)}(z, m ; p, q), \\
\tilde{\Gamma}^{(r)}(z, m+r ; p, q)=\tilde{\Gamma}^{(r)}(z, m ; p, q),
\end{gathered}
$$

for rewriting the sum-integrals without employing the $\llbracket m \rrbracket$-function. Note that our normalization factor coincides with the above one for $r=1$. Clearly, this is a multivalued function of its arguments and a particular branch of the power of $-1=e^{-\pi \mathrm{i}}$ was chosen in the considerations of [23]. Therefore the sum-integrals obtained after replacing our $\Gamma^{(r)}(z, m ; p, q)$ by (62) will be multivalued as well. Still, the balancing condition removes this drawback for the integration variable $z$ and, as claimed in [23], the $\epsilon=0$ rarefied elliptic beta integral evaluation formula written in terms of (62) yields exactly the same identity as derived in the present paper. The author checked that this is true for $\epsilon \neq 0$ as well.

Due to the periodicity, for sum-integrals written in terms of function (62) the discrete balancing condition takes the form $\sum_{a=1}^{6}\left(n_{a}+\epsilon / 2\right)=K r$, for arbitrary $K \in \mathbb{Z}$. Using the quasiperiodicity of the $\gamma^{(r)}(z, m ; p, q)$-function it is possible to remove this integer $K$ by a simple shift $n_{a} \rightarrow n_{a}+K r$ for any fixed $a$. Therefore our balancing condition 
(corresponding to $K=0$ ) is a generic one. As a result, the approaches developed in this paper and the one of [23, 32] are equivalent. An advantage of the present consideration is that all our sum-integrals are single-valued functions of parameters, which is not so in the other case.

Let us stress that the choice of the normalization factor leading to the periodicity (63) is not unique. If we flip the minus sign from $\sqrt{p / q}$ to $\sqrt{p q}$, i.e. replace in (62) $e^{-\pi \mathrm{i} \frac{m(m-r)(2 m-r)}{6 r}}$ by $e^{-\pi \mathrm{i} \frac{m(m-r)}{2 r}}$, then the coincidence with our results breaks down, since this will bring a nontrivial $m$-dependent multivalued factor inside the summation $\sum_{m=0}^{r-1}$. So, not all periodic gamma functions work equally well. It is necessary to understand the reasons distinguishing the choice (62).

Assume now that $r>1$ and consider the simplest $p \rightarrow 0$ limit in the above relation (61) for a special choice of discrete parameters

$$
n_{1,2,3}=0, \quad n_{4,5,6}=-\epsilon .
$$

For that we substitute into the arguments of $\gamma^{(r)}$-functions the relation $t_{6}=p q / \prod_{a=1}^{5} t_{a}$ and use the inversion relation (22). Now we can take the limit $p \rightarrow 0$ for fixed $t_{1}, \ldots, t_{5}$. Whenever the modulus of discrete arguments of the $\gamma^{(r)}$-functions is bounded by $r$, we use the asymptotic relation

$$
\gamma^{(r)}(z, m ; p, q) \underset{p \rightarrow 0}{\rightarrow}\left\{\begin{array}{cl}
\left(z q^{r-m} ; q^{r}\right)_{\infty}^{-1}, & 0<m \leq r, \\
\left(z ; q^{r}\right)_{\infty}^{-1}, & m=0, \\
\left(\frac{-p}{z}\right)^{|m| \mid} \frac{(p / q)^{|m|(|m|-1) / 2}}{\left(z q^{|m|} ; q^{r}\right)_{\infty}}, & -r<m<0 .
\end{array}\right.
$$

For other values of $m$ we apply first the quasiperiodicity relation (21) before taking the asymptotics. Because of the symmetry $c_{r-m}=c_{m-\epsilon}$, it is sufficient to consider the asymptotics of the coefficients $c_{0}, \ldots, c_{\ell}$ with $\ell=r / 2$ (for even $r$ ) or $\ell=(r-1) / 2$ (for odd $r$ ), provided $\epsilon=0$. For $\epsilon=1$ one has either $\ell=r / 2-1$ (for even $r$ ) or $\ell=(r-1) / 2$ (for odd $r$ ), see (42) and (43). Combining all asymptotic terms we obtain the following picture.

For $\epsilon=0$ and $m=0, \ldots,[r / 2]$ (an integral part of $r / 2$ ) the coefficients $c_{m} \propto p^{m-1 / 4}, p \rightarrow$ 0 for both even and odd $r$. As a result, in the limit of interest the leading asymptotics of $c_{m}$ corresponds to $m=0$. After considering the leading asymptotics of the right-hand side expression in (61), we come to the following identity (recall that $\left|t_{a}\right|<1$ )

$$
\frac{\left(q^{r} ; q^{r}\right)_{\infty}}{4 \pi \mathrm{i}} \int_{\mathbb{T}} \frac{\left(\prod_{b=1}^{5} t_{b} z^{ \pm 1} ; q^{r}\right)_{\infty}\left(z^{ \pm 2} ; q^{r}\right)_{\infty}}{\prod_{a=1}^{5}\left(t_{a} z^{ \pm 1} ; q^{r}\right)_{\infty}} \frac{d z}{z}=\frac{\prod_{a=1}^{5}\left(t_{a}^{-1} \prod_{b=1}^{5} t_{b} ; q^{r}\right)_{\infty}}{\prod_{1 \leq a<b \leq 5}\left(t_{a} t_{b} ; q^{r}\right)_{\infty}},
$$

which is the Rahman $q$-beta integral evaluation formula [34] for the base $q^{r}$. We conclude that in this case the rarefied elliptic beta integral does not produce new objects.

However, for $\epsilon=1$ the situation is qualitatively different. First we note that in this case our choice of the discrete variables $n_{a}$ breaks $S_{6}$-symmetry. Therefore the consideration depends on whether we take $t_{6} \rightarrow 0$ as $p \rightarrow 0$ (as above), or if one of $t_{a}, a=1,2,3$, tends to zero. Keeping $t_{1}, \ldots, t_{5}$ fixed we repeat the same steps as above. Then for even $r$ we find the asymptotics $c_{m} \propto p^{m+3 / 4}, p \rightarrow 0$ with $m=0, \ldots, r / 2-1$. For odd $r$ we find $c_{m} \propto p^{m+3 / 4}, p \rightarrow 0$ with $m=0, \ldots,(r-1) / 2$. In both cases the leading 
term corresponds to $m=0$. As follows from (43) such a term enters twice the full sum over $m$. Computing asymptotics of the right-hand side expression, we come to the same leading behaviour. After cancelling diverging factors there emerges a nontrivial relation. Omitting the technical details of computations for both sides of the identity (61), we come to the following statement.

Theorem 4. Let $r \in \mathbb{Z}_{>1}$ and the variables $t_{1}, \ldots, t_{5}, q \in \mathbb{C}$ are such that $\left|t_{a}\right|,|q|<1$. Then

$$
\begin{aligned}
& \frac{\left(q^{r} ; q^{r}\right)_{\infty}}{2 \pi \mathrm{i}} \int_{\mathbb{T}} \frac{\left(q^{r-1} A z, A z^{-1}, q^{r-1} z^{2}, q z^{-2} ; q^{r}\right)_{\infty}}{\prod_{a=1}^{3}\left(q^{r-1} t_{a} z, t_{a} z^{-1} ; q^{r}\right)_{\infty} \prod_{a=4}^{5}\left(t_{a} z, q t_{a} z^{-1} ; q^{r}\right)_{\infty}} \frac{d z}{z} \\
& \quad=\frac{\prod_{a=1}^{3}\left(A t_{a}^{-1} ; q^{r}\right)_{\infty} \prod_{a=4}^{5}\left(q^{r-1} A t_{a}^{-1} ; q^{r}\right)_{\infty}}{\prod_{1 \leq a<b \leq 3}\left(q^{r-1} t_{a} t_{b} ; q^{r}\right)_{\infty} \prod_{a=1}^{3} \prod_{b=4}^{5}\left(t_{a} t_{b} ; q^{r}\right)_{\infty}\left(q t_{4} t_{5} ; q^{r}\right)_{\infty}}, \quad A=\prod_{a=1}^{5} t_{a} .
\end{aligned}
$$

Choosing $n_{1,2,3}=-\epsilon$ and $n_{4,5,6}=0$ and repeating the same limiting procedure, we find again relation (66) with flipped multipliers $q$ and $q^{r-1}$ in the arguments of $q$-shifted factorials. The derived formulae represent a new layer of $q$-hypergeometric identities which was not considered in the $q$-treatise [25]. It is distinguished by the breaking of $S_{5}$ symmetry of (65) and, especially, of the $z \rightarrow z^{-1}$ symmetry of the integrand, which is quite unusual. Note that the restriction $r>1$ in (66) comes from the fact that for $r=1$ the original relation (61) reduces to the standard elliptic beta integral which does not yield new relations in the $p \rightarrow 0$ limit.

Let us denote $t_{a}=q^{r u_{a}}, A=q^{r U}$, and take the limit $q \rightarrow 1^{-}$in (66) for fixed $u_{a}$. Using the uniform limiting relation for the Jackson $q$-gamma function [2, 25]

$$
\Gamma_{q}(x):=\frac{(q ; q)_{\infty}}{\left(q^{x} ; q\right)_{\infty}}(1-q)^{1-x} \underset{q \rightarrow 1^{-}}{\rightarrow} \Gamma(x),
$$

where $\Gamma(x)$ is the standard Euler gamma function, we come to the following statement.

Theorem 5. Let $r \in \mathbb{Z}_{>1}$ and the variables $u_{a} \in \mathbb{C}, a=1, \ldots, 5$, are such that $\operatorname{Re}\left(u_{a}\right)>0$. Then the following plain hypergeometric integral evaluation holds true

$$
\begin{array}{r}
\frac{1}{2 \pi \mathrm{i}} \int_{-\mathrm{i} \infty}^{\mathrm{i} \infty} \frac{\prod_{a=1}^{3} \Gamma\left(\frac{r-1}{r}+u_{a}+x, u_{a}-x\right) \prod_{a=4}^{5} \Gamma\left(u_{a}+x, \frac{1}{r}+u_{a}-x\right)}{\Gamma\left(\frac{r-1}{r}+U+x, U-x, \frac{r-1}{r}+2 x, \frac{1}{r}-2 x\right)} d x \\
=\frac{\prod_{1 \leq a<b \leq 3} \Gamma\left(\frac{r-1}{r}+u_{a}+u_{b}\right) \prod_{a=1}^{3} \prod_{b=4}^{5} \Gamma\left(u_{a}+u_{b}\right) \Gamma\left(\frac{1}{r}+u_{4}+u_{5}\right)}{\prod_{a=1}^{3} \Gamma\left(U-u_{a}\right) \prod_{a=4}^{5} \Gamma\left(\frac{r-1}{r}+U-u_{a}\right)},
\end{array}
$$

where $U=\sum_{a=1}^{5} u_{a}$.

A similar formula is obtained from (66) after flipping $q$ and $q^{r-1}$ - it corresponds to flipping $1 / r$ and $(r-1) / r$ in the arguments of gamma functions of (67).

Probably for other admissible values of discrete variables $n_{a}$ the limit $p \rightarrow 0$ produces other relations similar to (66) containing powers $q^{k}, k=2, \ldots, r-2$. We do not consider such possibilities here, since the derived result requires a systematic consideration of not 
only all possible exact integral evaluations emerging in this way or symmetry transformations (which we skip), but an investigation of the question what kind of orthogonal polynomials and biorthogonal rational functions correspond to the measures described by the above exactly computable $q$ - and ordinary hypergeometric beta integrals and their multivariate extensions. We conclude that the rarefied elliptic hypergeometric functions contain intriguingly new phenomena at the lower hypergeometric levels.

\section{A $C_{n}$ RAREFied ElLiptic Beta integral of TyPe II}

A rarefied analogue of the computable type II $C_{n}$-integral of [13] has the following form. For convenience we denote the rank of the root system (and the dimension of the related integral) as $d$, i.e. we consider the root system $C_{d}$.

Theorem 6. Let nine continuous parameters $t, t_{a}(a=1, \ldots, 6), p, q \in \mathbb{C}^{*}$ and eight discrete variables $n, n_{a} \in \mathbb{Z}, \epsilon=0,1$, satisfy the constraints $|p|,|q|,|t|,\left|t_{a}\right|<1$ and the balancing condition

$$
t^{2 d-2} \prod_{a=1}^{6} t_{a}=p q, \quad 2 n(d-1)+\sum_{a=1}^{6} n_{a}+3 \epsilon=0 .
$$

Then

$$
\begin{gathered}
\kappa_{d}^{(r)} \sum_{m_{1}, \ldots, m_{d}=0}^{r-1} \int_{\mathbb{T}^{d}} \prod_{1 \leq j<k \leq d} \frac{\Gamma\left(t z_{j}^{ \pm 1} z_{k}^{ \pm 1}, n \pm\left(m_{j}+\epsilon / 2\right) \pm\left(m_{k}+\epsilon / 2\right)\right)}{\Gamma\left(z_{j}^{ \pm 1} z_{k}^{ \pm 1}, \pm\left(m_{j}+\epsilon / 2\right) \pm\left(m_{k}+\epsilon / 2\right)\right)} \\
\times \prod_{j=1}^{d} \frac{\prod_{a=1}^{6} \Gamma\left(t_{a} z_{j}, n_{a}+m_{j}+\epsilon\right) \Gamma\left(t_{a} z_{j}^{-1}, n_{a}-m_{j}\right)}{\Gamma\left(z_{j}^{ \pm 2}, \pm\left(2 m_{j}+\epsilon\right)\right)} \frac{d z_{j}}{z_{j}} \\
=\prod_{j=1}^{d}\left(\frac{\Gamma\left(t^{j}, n j\right)}{\Gamma(t, n)} \prod_{1 \leq a<b \leq 6} \Gamma\left(t^{j-1} t_{a} t_{b}, n(j-1)+n_{a}+n_{b}+\epsilon\right)\right) .
\end{gathered}
$$

Proof. The general scheme of proving formula (69) will be the same as in the original considerations of [14]. We assume that the variables $t_{6}$ and $n_{6}$ are excluded with the help of the balancing condition and denote the expression on the left-hand side of (69) as 
$I_{d, \epsilon}^{(r)}\left(t, t_{1}, \ldots, t_{5}, n, n_{1}, \ldots, n_{5}\right)$. Consider now the following sum of $(2 d-1)$-tuple integrals

$$
\begin{aligned}
& \kappa_{d}^{(r)} \kappa_{d-1}^{(r)} \sum_{m_{1}, \ldots, m_{d}=0}^{r-1} \sum_{l_{1}, \ldots, l_{d-1}=0}^{r-1} \int_{\mathbb{T}^{2 d-1}} \prod_{j=1}^{d} \frac{d z_{j}}{z_{j}} \prod_{k=1}^{d-1} \frac{d w_{k}}{w_{k}} \\
& \times \prod_{1 \leq j<k \leq d} \frac{1}{\Gamma\left(z_{j}^{ \pm 1} z_{k}^{ \pm 1}, \pm\left(m_{j}+\epsilon / 2\right) \pm\left(m_{k}+\epsilon / 2\right)\right)} \\
& \times \prod_{j=1}^{n} \frac{\prod_{a=0}^{5} \Gamma\left(t_{a} z_{j}^{ \pm 1}, n_{a}+\frac{\epsilon}{2} \pm\left(m_{j}+\frac{\epsilon}{2}\right)\right)}{\Gamma\left(z_{j}^{ \pm 2}, \pm\left(2 m_{j}+\epsilon\right)\right)} \\
& \times \prod_{j=1}^{d} \prod_{k=1}^{d-1} \Gamma\left(t^{1 / 2} z_{j} w_{k}, n+\frac{\epsilon}{2}+\frac{\delta}{2} \pm\left(m_{j}+\frac{\epsilon}{2}\right) \pm\left(l_{k}+\frac{\delta}{2}\right)\right. \\
& \times \prod_{1 \leq j<k \leq d-1} \frac{d-1}{\Gamma\left(w_{j}^{ \pm 1} w_{k}^{ \pm 1}, \pm\left(l_{j}+\frac{\delta}{2}\right) \pm\left(l_{k}+\frac{\delta}{2}\right)\right)} \prod_{k=1} \frac{1}{\Gamma\left(w_{k}^{ \pm 2}, \pm\left(2 l_{k}+\delta\right)\right)} \\
& \times \prod_{k=1}^{d-1} \frac{\Gamma\left(w_{k}^{ \pm 1} t^{d-3 / 2} \prod_{a=1}^{5} t_{a},(2 d-3)\left(n+\frac{\epsilon}{2}+\frac{\delta}{2}\right)+\sum_{a=1}^{5}\left(n_{a}+\frac{\epsilon}{2}\right) \pm\left(l_{k}+\frac{\delta}{2}\right)\right)}{\Gamma\left(w_{k}^{ \pm 1} t^{2 d-3 / 2} \prod_{a=1}^{5} t_{a},(4 d-3)\left(n+\frac{\epsilon}{2}+\frac{\delta}{2}\right)+\sum_{a=1}^{5}\left(n_{a}+\frac{\epsilon}{2}\right) \pm\left(l_{k}+\frac{\delta}{2}\right)\right)}
\end{aligned}
$$

with $|t|,\left|t_{a}\right|<1(a=0, \ldots, 5), \epsilon=0,1, \delta=0,1$, and

$$
t^{d-1} \prod_{a=0}^{5} t_{a}=p q, \quad(d-1)(2 n+\epsilon+\delta)+\sum_{a=0}^{5} n_{a}+3 \epsilon=0
$$

Integration over the variables $w_{k}$ and summation over $l_{k}$ with the help of formula (46) brings expression (70) to the form

$$
\frac{\Gamma(t, 2 n+\epsilon+\delta)^{d}}{\Gamma\left(t^{d},(2 n+\epsilon+\delta) d\right)} I_{d, \epsilon}^{(r)}\left(t, t_{1}, \ldots, t_{5}, 2 n+\epsilon+\delta, n_{1}, \ldots, n_{5}\right)
$$

where the balancing condition (68) is assumed with $n$ replaced by $2 n+\epsilon+\delta$.

Because the integrand is bounded on the contour of integration, we can change the order of integrations. Then the integration over $z_{k}$-variables and summation over $m_{k}$ with the help of formula (46) converts expression (70) to

$$
\begin{aligned}
& \Gamma(t, 2 n+\epsilon+\delta)^{d-1} \prod_{0 \leq a<b \leq 5} \Gamma\left(t_{a} t_{b}, n_{a}+n_{b}+\epsilon\right) \\
& \quad \times I_{d-1, \delta}^{(r)}\left(t, t^{1 / 2} t_{1}, \ldots, t^{1 / 2} t_{5}, 2 n+\epsilon+\delta, n+n_{1}+\epsilon, \ldots, n+n_{5}+\epsilon\right) .
\end{aligned}
$$


As a result, we obtain a recurrence relation connecting $I_{d, \epsilon}^{(r)}$-functions of different rank $d$ and different $\epsilon$-variables:

$$
\begin{gathered}
\frac{I_{d, \epsilon}^{(r)}\left(t, t_{1}, \ldots, t_{5}, 2 n+\epsilon+\delta, n_{1}, \ldots, n_{5}\right)}{I_{d-1, \delta}^{(r)}\left(t, t^{1 / 2} t_{1}, \ldots, t^{1 / 2} t_{5}, 2 n+\epsilon+\delta, n+n_{1}+\epsilon, \ldots, n+n_{5}+\epsilon\right)} \\
=\frac{\Gamma\left(t^{d},(2 n+\epsilon+\delta) d\right)}{\Gamma(t, 2 n+\epsilon+\delta)} \prod_{0 \leq a<b \leq 5} \Gamma\left(t_{a} t_{b}, n_{a}+n_{b}+\epsilon\right) .
\end{gathered}
$$

For different choices of $\epsilon$ and $\delta$ one can reach both even and odd values of the sum $2 n+\epsilon+\delta$, which can be redenoted as an arbitrary integer $n \in \mathbb{Z}$. Then, using known initial condition for $d=1$ (41), we find (69) by recursion.

Evidently, for $d=1$ both multiple integrals (46) and (69) reduce to the rarefied elliptic beta integral (41). The relation (69) represents currently the most complicated known generalization of the Selberg integral along the lines introduced by Gustafson [26].

\section{An AnAlogue of the Euler-Gauss hypergeometric FUnCtion}

For the same values of the discrete variables $\epsilon$ and $\delta$ as in the previous section, consider the following double sum of double integrals

$$
\begin{aligned}
& \sum_{m=0}^{r-1} \sum_{l=0}^{r-1} \int_{\mathbb{T}^{2}} \Gamma\left(f z^{ \pm 1} w^{ \pm 1}, h+\frac{\epsilon}{2}+\frac{\delta}{2} \pm\left(m+\frac{\epsilon}{2}\right) \pm\left(l+\frac{\delta}{2}\right)\right) \\
& \quad \times \frac{\prod_{a=1}^{4} \Gamma\left(t_{a} z^{ \pm 1}, n_{a}+\frac{\epsilon}{2} \pm\left(m+\frac{\epsilon}{2}\right)\right) \Gamma\left(s_{a} w^{ \pm 1}, k_{a}+\frac{\delta}{2} \pm\left(l+\frac{\delta}{2}\right)\right)}{\Gamma\left(z^{ \pm 2}, \pm(2 m+\epsilon)\right) \Gamma\left(w^{ \pm 2}, \pm(2 l+\delta)\right)} \frac{d z}{z} \frac{d w}{w}
\end{aligned}
$$

where the variables $n_{a}, k_{a}, h \in \mathbb{Z}$ and $t_{a}, s_{a}, f \in \mathbb{C}^{*}$ satisfy the constraints $\left|t_{a}\right|,\left|s_{a}\right|,|f|,<1$ and the balancing conditions

$$
f^{2} \prod_{a=1}^{4} t_{a}=f^{2} \prod_{a=1}^{4} s_{a}=p q, \quad 2 h+\epsilon+\delta=-\sum_{a=1}^{4}\left(n_{a}+\frac{\epsilon}{2}\right)=-\sum_{a=1}^{4}\left(k_{a}+\frac{\delta}{2}\right) .
$$

We see that for different values of $\epsilon$ and $\delta$, the sums $\sum_{a=1}^{4} n_{a}$ and $\sum_{a=1}^{4} k_{a}$ can be both (simultaneously) odd or even integers.

Because of the imposed constraints, the contour $\mathbb{T}$ is legitimate for computing sumintegrals over $(z, m)$ or $(w, \ell)$ with the help of formula (41). Integrate first over $z$ and sum over $m$. Then, using Fubini's theorem, we change the order and integrate first over 
$w$ and sum over $l$. This yields the following identity.

$$
\begin{aligned}
\sum_{l=0}^{r-1} & \int_{\mathbb{T}} \frac{\prod_{a=1}^{4} \Gamma\left(f t_{a} w^{ \pm 1}, h+n_{a}+\epsilon+\frac{\delta}{2} \pm\left(l+\frac{\delta}{2}\right)\right) \Gamma\left(s_{a} w^{ \pm 1}, k_{a}+\frac{\delta}{2} \pm\left(l+\frac{\delta}{2}\right)\right)}{\Gamma\left(w^{ \pm 2}, \pm(2 l+\delta)\right)} \frac{d w}{w} \\
= & \prod_{1 \leq a<b \leq 4} \frac{\Gamma\left(s_{a} s_{b}, k_{a}+k_{b}+\delta\right)}{\Gamma\left(t_{a} t_{b}, n_{a}+n_{b}+\epsilon\right)} \\
& \times \sum_{m=0}^{r-1} \int_{\mathbb{T}} \frac{\prod_{a=1}^{4} \Gamma\left(t_{a} z^{ \pm 1}, n_{a}+\frac{\epsilon}{2} \pm\left(m+\frac{\epsilon}{2}\right)\right) \Gamma\left(f s_{a} z^{ \pm 1}, h+k_{a}+\delta+\frac{\epsilon}{2} \pm\left(m+\frac{\epsilon}{2}\right)\right)}{\Gamma\left(z^{ \pm 2}, \pm(2 m+\epsilon)\right)} \frac{d z}{z} .
\end{aligned}
$$

Let us define the rarefied elliptic hypergeometric function

$$
V_{\epsilon}^{(r)}\left(t_{a}, n_{a} ; p, q\right)=\kappa^{(r)} \sum_{m=0}^{r-1} \int_{\mathbb{T}} \frac{\prod_{a=1}^{8} \Gamma\left(t_{a} z, n_{a}+\epsilon+m\right) \Gamma\left(t_{a} z^{-1}, n_{a}-m\right)}{\Gamma\left(z^{ \pm 2}, \pm(2 m+\epsilon)\right)} \frac{d z}{z},
$$

where $t_{a} \in \mathbb{C}^{*},\left|t_{a}\right|<1, n_{a} \in \mathbb{Z}, \epsilon=0,1$, and

$$
\prod_{a=1}^{8} t_{a}=(p q)^{2}, \quad \sum_{a=1}^{8} n_{a}+4 \epsilon=0 .
$$

As usual, the contour $\mathbb{T}$ separates geometric progressions of poles converging to zero from their partners going to infinity. Other domains of values of the parameters are reached by analytic continuation. Both, the function itself (73) and the balancing condition (74), are invariant with respect to the group $S_{8}$ permuting parameters $t_{a}$ and $n_{a}$ (which is the Weyl group of the root system $A_{7}$ ). For $r=1$ this is the elliptic analogue of the Euler-Gauss hypergeometric function introduced in [44], $V_{\epsilon}^{(1)}\left(t_{a}, n_{a} ; p, q\right) \equiv V\left(t_{a} ; p, q\right)$.

Suppose that parameters $t_{7}, t_{8}, n_{7}$, and $n_{8}$ satisfy the constraints $t_{7} t_{8}=p q$ and $n_{7}+$ $n_{8}+\epsilon=0$. Then we have

$$
\Gamma^{(r)}\left(t_{7} z, n_{7}+m+\epsilon\right) \Gamma^{(r)}\left(t_{8} z^{-1}, n_{8}-m\right)=1
$$

and these parameters drop out completely from the $V^{(r)}$-function, which thus becomes equal to the rarefied elliptic beta integral.

Quasiperiodicity of the rarefied elliptic gamma function leads to the relation

$$
\begin{aligned}
& V_{\epsilon}^{(r)}\left(\ldots, n_{b}+r, \ldots, n_{c}-r, \ldots ; p, q\right)=V_{\epsilon}^{(r)}\left(t_{a}, n_{a} ; p, q\right) \\
& \quad \times\left[t_{b}^{r+2 n_{b}+\epsilon} t_{c}^{r-2 n_{c}-\epsilon}\left(p^{1-n_{b}-n_{c}-\epsilon} q^{1+n_{b}+n_{c}+\epsilon}\right)^{n_{c}-n_{b}-r}\right]^{r-1} .
\end{aligned}
$$

For odd $r$ this relation allows one to convert all $n_{a}$ to even numbers, i.e. without loss of generality, for odd $r$ we can assume that all $n_{a}$ are even.

Substituting definition (73) into relation (72), we obtain the transformation property of the $V_{\epsilon}^{(r)}$-function

$$
\begin{aligned}
V_{\epsilon}^{(r)}\left(t_{a},\right. & \left.n_{a} ; p, q\right)=V_{\delta}^{(r)}\left(s_{a}, k_{a} ; p, q\right) \\
& \times \prod_{1 \leq b<c \leq 4} \Gamma\left(t_{b} t_{c}, n_{b}+n_{c}+\epsilon\right) \Gamma\left(t_{b+4} t_{c+4}, n_{b+4}+n_{c+4}+\epsilon\right),
\end{aligned}
$$


where

$$
\begin{aligned}
& \left\{\begin{array}{cc}
s_{a}=f t_{a}, & a=1,2,3,4, \\
s_{a}=f^{-1} t_{a}, & a=5,6,7,8,
\end{array} ; \quad f=\sqrt{\frac{p q}{t_{1} t_{2} t_{3} t_{4}}}=\sqrt{\frac{t_{5} t_{6} t_{7} t_{8}}{p q}},\right. \\
& \begin{cases}k_{a}=n_{a}-\frac{1}{2}\left(\sum_{b=1}^{4} n_{b}+\epsilon+\delta\right), & a=1,2,3,4, \\
k_{a}=n_{a}-\frac{1}{2}\left(\sum_{b=5}^{8} n_{b}+\epsilon+\delta\right), & a=5,6,7,8,\end{cases}
\end{aligned}
$$

and is it assumed that $\left|t_{a}\right|,\left|s_{a}\right|<1$. In the space of continuous parameters $t_{a}$, the map $t_{a} \rightarrow s_{a}$ is the key reflection transformation extending $S_{8}$ to $W\left(E_{7}\right)$, the Weyl group of the exceptional root system $E_{7}$, in the same way as in the $r=1$ case.

However, in the space of discrete variables $n_{a}$ the situation is more complicated. The function $V_{\epsilon}^{(r)}$ does not depend on $\delta$, which has the appearance of a free parameter. However, this is not true - the above transformation is meaningful only when $\sum_{b=1}^{4} n_{b}+\epsilon+\delta$ is an even integer, and it is from this condition that the value of $\delta$ is determined. So, for even or odd $\sum_{b=1}^{4} n_{b}+\epsilon$ one should take $\delta=0$ or $\delta=1$, respectively. As mentioned above, for odd $r$ all $n_{a}$ can be taken even, and in this case one has $\delta=\epsilon$. The same situation holds when all $n_{a}$ are odd. However, when $r$ is even and variables $n_{a}$ take both odd and even integer values, the transformation law of discrete variables does not look $S_{8}$ symmetric, and $\delta \neq \epsilon$ cases are allowed. In terms of the variables $n_{a}^{\prime}=n_{a}+\epsilon / 2$ and $k_{a}^{\prime}=k_{a}+\delta / 2$ the transformation (176) takes the standard reflection form

$$
k_{a}^{\prime}=n_{a}^{\prime}-\frac{1}{2} \sum_{b=1}^{4} n_{b}^{\prime}, a=1,2,3,4, \quad k_{a}^{\prime}=n_{a}^{\prime}-\frac{1}{2} \sum_{b=5}^{8} n_{b}^{\prime}, a=5,6,7,8 .
$$

Note that $n_{a}^{\prime}$ and $k_{a}^{\prime}$ may be not integer valued and the choice of $\delta$ depends on $n_{a}$. Therefore, the action of full $W\left(E_{7}\right)$ symmetry on discrete variables $n_{a}$ gets a curious deformed form. The detailed analysis of this phenomenon lies beyond the scope of this work.

Similar to the standard $V$-function situation, there are two more distinguished forms of the $W\left(E_{7}\right)$-transformations. The second transformation is obtained after repeating (75) with $\delta$ playing the role of $\epsilon, s_{3,4,5,6}$ playing the role of $t_{1,2,3,4}$ and $k_{3,4,5,6}$ playing the role of $n_{1,2,3,4}$. Also, one has to introduce another discrete variable $\rho=0,1$, an analogue of $\delta$ in the first transformation. After symmetrization of the resulting relation, we obtain the identity:

$$
V_{\epsilon}^{(r)}\left(t_{a}, n_{a} ; p, q\right)=\prod_{1 \leq b, c \leq 4} \Gamma\left(t_{b} t_{c+4}, n_{b}+n_{c+4}+\epsilon\right) V_{\rho}^{(r)}\left(s_{a}, k_{a} ; p, q\right)
$$

where

$$
s_{a}=\frac{\sqrt{t_{1} t_{2} t_{3} t_{4}}}{t_{a}}, a=1,2,3,4, \quad s_{a}=\frac{\sqrt{t_{5} t_{6} t_{7} t_{8}}}{t_{a}}, a=5,6,7,8,
$$

with $\left|t_{a}\right|,\left|s_{a}\right|<1$ and

$$
\begin{cases}k_{a}=-n_{a}+\frac{1}{2}\left(\sum_{\ell=1}^{4} n_{\ell}+\epsilon-\rho\right), & a=1,2,3,4 \\ k_{a}=-n_{a}+\frac{1}{2}\left(\sum_{\ell=5}^{8} n_{\ell}+\epsilon-\rho\right), & a=5,6,7,8 .\end{cases}
$$

Here the value of $\rho$ is fixed from the condition that $\sum_{\ell=1}^{4} n_{\ell}+\epsilon-\rho$ is even. 
The third transformation is obtained after equating the right-hand side expressions in (75) and (77):

$$
V_{\epsilon}^{(r)}\left(t_{a}, n_{a} ; p, q\right)=\prod_{1 \leq b<c \leq 8} \Gamma\left(t_{b} t_{c}, n_{b}+n_{c}+\epsilon\right) V_{\epsilon}^{(r)}\left(\frac{\sqrt{p q}}{t_{a}},-n_{a}-\epsilon ; p, q\right) .
$$

Originally one finds in this case the transformation $n_{a} \rightarrow-n_{a}-(\epsilon+\delta) / 2$, which requires $\epsilon+\delta$ to be even leading to $\delta=\epsilon$. Note that the form of this third transformation is true for arbitrary values of $n_{a}$, since the map $n_{a} \rightarrow-n_{a}-\epsilon$ is true for all $n_{a}$.

For $r=1$ all three relations become the standard symmetry transformations for the $V$-function with the key generating relation (75) discovered in 44 .

Let us construct the rarefied analogue of the elliptic hypergeometric equation derived in [46, 48]. For brevity we use the following trick - until formula (82) the symbols $n_{a}$ and $m$ actually denote $n_{a}+\epsilon / 2$ and $m+\epsilon / 2$. This is legitimate, since the integrality of $n_{a}$ and $m$ is not essential in the computations. However, to remind on the $\epsilon$-dependence we keep the notation $V_{\epsilon}^{(r)}$.

The addition formula for elliptic theta functions can be written in the form

$$
t_{3} \theta\left(t_{2} t_{3}^{ \pm 1}, t_{1} z^{ \pm 1} ; q^{r}\right)+t_{1} \theta\left(t_{3} t_{1}^{ \pm 1}, t_{2} z^{ \pm 1} ; q^{r}\right)+t_{2} \theta\left(t_{1} t_{2}^{ \pm 1}, t_{3} z^{ \pm 1} ; q^{r}\right)=0 .
$$

It yields the following contiguous relation for the $V_{\epsilon}^{(r)}$-function

$$
\begin{gathered}
\frac{t_{1}^{1+2 n_{1}} q^{-n_{1}\left(n_{1}+2\right)} V_{\epsilon}^{(r)}\left(p t_{1}, n_{1}-1\right)}{\theta\left(t_{1} t_{2}^{ \pm 1} q^{-n_{1} \mp n_{2}}, t_{1} t_{3}^{ \pm 1} q^{-n_{1} \mp n_{3}} ; q^{r}\right)}+\frac{t_{2}^{1+2 n_{2}} q^{-n_{2}\left(n_{2}+2\right)} V_{\epsilon}^{(r)}\left(p t_{2}, n_{2}-1\right)}{\theta\left(t_{2} t_{1}^{ \pm 1} q^{-n_{2} \mp n_{1}}, t_{2} t_{3}^{ \pm 1} q^{-n_{2} \mp n_{3}} ; q^{r}\right)} \\
+\frac{t_{3}^{1+2 n_{3}} q^{-n_{3}\left(n_{3}+2\right)} V_{\epsilon}^{(r)}\left(p t_{3}, n_{3}-1\right)}{\theta\left(t_{3} t_{1}^{ \pm 1} q^{-n_{3} \mp n_{1}}, t_{3} t_{2}^{ \pm 1} q^{-n_{3} \mp n_{2}} ; q^{r}\right)}=0,
\end{gathered}
$$

where $V_{\epsilon}^{(r)}\left(p t_{b}, n_{b}-1\right)$ denotes the $V_{\epsilon}^{(r)}\left(t_{a}, n_{a}\right)$-function with the parameters $t_{b}, n_{b}$ replaced by $p t_{b}, n_{b}-1$ (with the balancing condition being $\prod_{a=1}^{8} t_{a}=p q^{2}, \sum_{a=1}^{8} n_{a}=1$ ). Indeed, if we replace in (80) $V_{\epsilon}^{(r)}$-functions by their integrands, then we obtain the equality

$$
\begin{gathered}
\frac{t_{1} q^{-n_{1}} \theta\left(t_{1} z^{ \pm 1} q^{-n_{1} \mp m} ; q^{r}\right)}{\theta\left(t_{1} t_{2}^{ \pm 1} q^{-n_{1} \mp n_{2}}, t_{1} t_{3}^{ \pm 1} q^{-n_{1} \mp n_{3}} ; q^{r}\right)}+\frac{t_{2} q^{-n_{2}} \theta\left(t_{2} z^{ \pm 1} q^{-n_{2} \mp m} ; q^{r}\right)}{\theta\left(t_{2} t_{1}^{ \pm 1} q^{-n_{2} \mp n_{1}}, t_{2} t_{3}^{ \pm 1} q^{-n_{2} \mp n_{3}} ; q^{r}\right)} \\
+\frac{t_{3} q^{-n_{3}} \theta\left(t_{3} z^{ \pm 1} q^{-n_{3} \mp m} ; q^{r}\right)}{\theta\left(t_{3} t_{1}^{ \pm 1} q^{-n_{3} \mp n_{1}}, t_{3} t_{2}^{ \pm 1} q^{-n_{3} \mp n_{2}} ; q^{r}\right)}=0
\end{gathered}
$$

multiplied by the function

$$
z^{2 m} q^{m^{2}} \prod_{a=1}^{8} \frac{\Gamma\left(t_{a} z^{ \pm 1}, n_{a} \pm m\right)}{\Gamma\left(z^{ \pm 2}, \pm 2 m\right)}
$$

Replacing $t_{a} \rightarrow t_{a} q^{n_{a}}$ and $z \rightarrow z q^{m}$ and simplifying the factors we obtain the addition formula (79). Integrating the resulting equation for the integrand functions over $z \in \mathbb{T}$ and summing in $m$, we come to (80). Note that for $r=1$ one can pull out all powers of $q$ out of the theta functions and find that all three terms in (80) get equal multipliers 
$q^{\sum_{a=1}^{3} n_{a}^{2}} / \prod_{a=1}^{3} t_{a}^{2 n_{a}}$, so that the dependence of this contiguous relation on $n_{a}$ disappears completely.

Substituting relation (78) in (80), we obtain

$$
\begin{aligned}
& \frac{p^{2 n_{1}} q^{n_{1}\left(n_{1}+3\right)}}{t_{1}^{2+2 n_{1}}} \frac{\prod_{a=4}^{8} \theta\left(\frac{t_{1} t_{a}}{p q} q^{-n_{1}-n_{a}} ; q^{r}\right)}{\theta\left(\frac{t_{2}}{t_{1}} q^{n_{1}-n_{2}}, \frac{t_{3}}{t_{1}} q^{n_{1}-n_{3}} ; q^{r}\right)} V_{\epsilon}^{(r)}\left(p^{-1} t_{1}, n_{1}+1\right) \\
& +\frac{p^{2 n_{2}} q^{n_{2}\left(n_{2}+3\right)}}{t_{2}^{2+2 n_{2}}} \frac{\prod_{a=4}^{8} \theta\left(\frac{t_{2} t_{a}}{p q} q^{-n_{2}-n_{a}} ; q^{r}\right)}{\theta\left(\frac{t_{1}}{t_{2}} q^{n_{2}-n_{1}}, \frac{t_{3}}{t_{2}} q^{n_{2}-n_{3}} ; q^{r}\right)} V_{\epsilon}^{(r)}\left(p^{-1} t_{2}, n_{2}+1\right) \\
& +\frac{p^{2 n_{3}} q^{n_{3}\left(n_{3}+3\right)}}{t_{3}^{2+2 n_{3}}} \frac{\prod_{a=4}^{8} \theta\left(\frac{t_{3} t_{a}}{p q} q^{-n_{3}-n_{a}} ; q^{r}\right)}{\theta\left(\frac{t_{2}}{t_{3}} q^{n_{3}-n_{2}}, \frac{t_{1}}{t_{3}} q^{n_{3}-n_{1}} ; q^{r}\right)} V_{\epsilon}^{(r)}\left(p^{-1} t_{3}, n_{3}+1\right)=0,
\end{aligned}
$$

where $\prod_{a=1}^{8} t_{a}=p^{3} q^{2}$ and $\sum_{a=1}^{8} n_{a}=-1$. Shifting $t_{3} \rightarrow p t_{3}$ and $n_{3} \rightarrow n_{3}-1$ in (81), we come to the equality

$$
\begin{aligned}
& \frac{p^{2 n_{1}} q^{n_{1}\left(n_{1}+3\right)}}{t_{1}^{2+2 n_{1}}} \frac{\prod_{a=4}^{8} \theta\left(\frac{t_{1} t_{a}}{p q} q^{-n_{1}-n_{a}} ; q^{r}\right)}{\theta\left(\frac{t_{2}}{t_{1}} q^{n_{1}-n_{2}}, \frac{p q t_{3}}{t_{1}} q^{n_{1}-n_{3}} ; q^{r}\right)} V_{\epsilon}^{(r)}\left(p^{-1} t_{1}, p t_{3}, n_{1}+1, n_{3}-1\right) \\
& +\frac{p^{2 n_{2}} q^{n_{2}\left(n_{2}+3\right)}}{t_{2}^{2+2 n_{2}}} \frac{\prod_{a=4}^{8} \theta\left(\frac{t_{2} t_{a}}{p q} q^{-n_{2}-n_{a}} ; q^{r}\right)}{\theta\left(\frac{t_{1}}{t_{2}} q^{n_{2}-n_{1}}, \frac{p q t_{3}}{t_{2}} q^{n_{2}-n_{3}} ; q^{r}\right)} V_{\epsilon}^{(r)}\left(p^{-1} t_{2}, p t_{3}, n_{2}+1, n_{3}-1\right) \\
& +\frac{p^{2\left(n_{3}-1\right)} q^{\left(n_{3}-1\right)\left(n_{3}+2\right)}}{\left(p t_{3}\right)^{2 n_{3}}} \frac{\prod_{a=4}^{8} \theta\left(t_{3} t_{a} q^{-n_{3}-n_{a}} ; q^{r}\right)}{\theta\left(\frac{t_{2}}{p q t_{3}} q^{n_{3}-n_{1}}, \frac{t_{1}}{p q t_{3}} q^{n_{3}-n_{1}} ; q^{r}\right)} V_{\epsilon}^{(r)}\left(t_{a}, n_{a}\right)=0 .
\end{aligned}
$$

Replacing $t_{1} \rightarrow p^{-1} t_{1}, n_{1} \rightarrow n_{1}+1$ or $t_{2} \rightarrow p^{-1} t_{2}, n_{2} \rightarrow n_{2}+1$ in (80) we obtain the relations

$$
\begin{aligned}
& \frac{\left(p^{-1} t_{1}\right)^{3+2 n_{1}} q^{-\left(n_{1}+1\right)\left(n_{1}+3\right)} V_{\epsilon}^{(r)}\left(t_{a}, n_{a}\right)}{\theta\left(p^{-1} t_{1} t_{2}^{ \pm 1} q^{-n_{1}-1 \mp n_{2}}, p^{-1} t_{1} t_{3}^{ \pm 1} q^{-n_{1}-1 \mp n_{3}} ; q^{r}\right)} \\
& +\frac{t_{2}^{1+2 n_{2}} q^{-n_{2}\left(n_{2}+2\right)} V_{\epsilon}^{(r)}\left(p^{-1} t_{1}, p t_{2}, n_{1}+1, n_{2}-1\right)}{\theta\left(t_{2}\left(p^{-1} t_{1}\right)^{ \pm 1} q^{-n_{2} \mp\left(n_{1}+1\right)}, t_{2} t_{3}^{ \pm 1} q^{-n_{2} \mp n_{3}} ; q^{r}\right)} \\
& \quad+\frac{t_{3}^{1+2 n_{3}} q^{-n_{3}\left(n_{3}+2\right)} V_{\epsilon}^{(r)}\left(p^{-1} t_{1}, p t_{3}, n_{1}+1, n_{3}-1\right)}{\theta\left(t_{3}\left(p^{-1} t_{1}\right)^{ \pm 1} q^{-n_{3} \mp\left(n_{1}+1\right)}, t_{3} t_{2}^{ \pm 1} q^{-n_{3} \mp n_{2}} ; q^{r}\right)}=0
\end{aligned}
$$


or

$$
\begin{aligned}
& \frac{t_{1}^{1+2 n_{1}} q^{-n_{1}\left(n_{1}+2\right)} V_{\epsilon}^{(r)}\left(p t_{1}, p^{-1} t_{2}, n_{1}-1, n_{2}+1\right)}{\theta\left(t_{1}\left(p^{-1} t_{2}\right)^{ \pm 1} q^{-n_{1} \mp\left(n_{2}+1\right)}, t_{1} t_{3}^{ \pm 1} q^{-n_{1} \mp n_{3}} ; q^{r}\right)} \\
& \quad+\frac{\left(p^{-1} t_{2}\right)^{3+2 n_{2}} q^{-\left(n_{2}+1\right)\left(n_{2}+3\right)} V_{\epsilon}^{(r)}\left(t_{a}, n_{a}\right)}{\theta\left(p^{-1} t_{2} t_{1}^{ \pm 1} q^{-n_{2}-1 \mp n_{1}}, p^{-1} t_{2} t_{3}^{ \pm 1} q^{-n_{2}-1 \mp n_{3}} ; q^{r}\right)} \\
& \quad+\frac{t_{3}^{1+2 n_{3}} q^{-n_{3}\left(n_{3}+2\right)} V_{\epsilon}^{(r)}\left(p^{-1} t_{2}, p t_{3}, n_{2}+1, n_{3}-1\right)}{\theta\left(t_{3} t_{1}^{ \pm 1} q^{-n_{3} \mp n_{1}}, t_{3}\left(p^{-1} t_{2}\right)^{ \pm 1} q^{-n_{3} \mp\left(n_{2}+1\right)} ; q^{r}\right)}=0 .
\end{aligned}
$$

Eliminating from the latter three equalities the functions $V_{\epsilon}^{(r)}\left(p^{-1} t_{1}, p t_{3}, n_{1}+1, n_{3}-1\right)$ and $V_{\epsilon}^{(r)}\left(p^{-1} t_{2}, p t_{3}, n_{2}+1, n_{3}-1\right)$, we arrive at the final equation (we restore the $\epsilon$-dependence):

$$
\begin{aligned}
& \mathcal{A}\left(\frac{t_{1}}{q^{n_{1}+\epsilon / 2}}, \frac{t_{2}}{q^{n_{2}+\epsilon / 2}}, \ldots, \frac{t_{8}}{q^{n_{8}+\epsilon / 2}}, p ; q^{r}\right)\left(U_{\epsilon}\left(p t_{1}, p^{-1} t_{2}, n_{1}-1, n_{2}+1\right)-U_{\epsilon}\left(t_{a}, n_{a}\right)\right) \\
& \quad+\mathcal{A}\left(\frac{t_{2}}{q^{n_{2}+\epsilon / 2}}, \frac{t_{1}}{q^{n_{1}+\epsilon / 2}}, \ldots, \frac{t_{8}}{q^{n_{8}+\epsilon / 2}}, p ; q^{r}\right)\left(U_{\epsilon}\left(p^{-1} t_{1}, p t_{2}, n_{1}+1, n_{2}-1\right)-U_{\epsilon}\left(t_{a}, n_{a}\right)\right) \\
& \quad+U_{\epsilon}\left(t_{a}, n_{a}\right)=0,
\end{aligned}
$$

where we have denoted

$$
\mathcal{A}\left(t_{1}, \ldots, t_{8}, p ; q^{r}\right):=\frac{\theta\left(\frac{t_{1}}{p q^{1-r} t_{3}}, t_{3} t_{1}, \frac{t_{3}}{t_{1}} ; q^{r}\right)}{\theta\left(\frac{t_{1}}{t_{2}}, \frac{t_{2}}{p q^{1-r} t_{1}}, \frac{t_{1} t_{2}}{p q^{1-r}} ; q^{r}\right)} \prod_{a=4}^{8} \frac{\theta\left(\frac{t_{2} t_{a}}{p q^{1-r}} ; q^{r}\right)}{\theta\left(t_{3} t_{a} ; q^{r}\right)}
$$

and

$$
U_{\epsilon}\left(t_{a}, n_{a}\right):=\frac{V_{\epsilon}^{(r)}\left(t_{a}, n_{a}\right)}{\prod_{k=1}^{2} \Gamma\left(t_{k} t_{3}, n_{k}+n_{3}+\epsilon\right) \Gamma\left(t_{k} t_{3}^{-1}, n_{k}-n_{3}\right)} .
$$

A fundamental fact is that for any $r$ the function $\mathcal{A}\left(t_{1}, \ldots, t_{8}, p ; q^{r}\right)$ is a $q^{r}$-elliptic function of all parameters $t_{1}, \ldots, t_{8}$ (one of which should be counted as a dependent variable through the balancing condition $\left.\prod_{a=1}^{8} t_{a}=(p q)^{2}\right)$, i.e. it does not change after the scaling $t_{a} \rightarrow t_{a} q^{r}, t_{b} \rightarrow t_{b} q^{-r}$ for any $a \neq b$.

We call equation (82) the rarefied elliptic hypergeometric equation, though it does not have the form one would have liked to see. It can be checked that under the shifts $n_{a} \rightarrow n_{a}+r, n_{b} \rightarrow n_{b}-r, a \neq b$ the functions $U_{\epsilon}\left(p^{ \pm 1} t_{1}, p^{\mp 1} t_{2}, n_{1} \mp 1, n_{2} \pm 1\right)$ and $U_{\epsilon}\left(t_{a}, n_{a}\right)$ have the same quasiperiodicity multipliers. Therefore, by shifting $t_{1,2} \rightarrow p^{ \pm l} t_{1,2}$, $n_{1,2} \rightarrow n_{1,2} \mp l, l=1,2, \ldots$, in equation (82), combining the resulting equation in an appropriate way and using the fact that

$$
U_{\epsilon}\left(t_{a}, n_{1}-r, n_{2}+r, n_{3}, \ldots\right)=U_{\epsilon}\left(t_{a}, n_{1}+r, n_{2}-r, n_{3}, \ldots\right)=U_{\epsilon}\left(t_{a}, n_{a}\right),
$$

one can derive the following tridiagonal equation

$$
\alpha\left(t_{a}, n_{a}\right) U_{\epsilon}\left(p^{r} t_{1}, p^{-r} t_{2}, n_{a}\right)+\beta\left(t_{a}, n_{a}\right) U_{\epsilon}\left(t_{a}, n_{a}\right)+\gamma\left(t_{a}, n_{a}\right) U_{\epsilon}\left(p^{-r} t_{1}, p^{r} t_{2}, n_{a}\right)=0,
$$

for some coefficients $\alpha, \beta, \gamma$. After parametrization $t_{1}=c x, t_{2}=c x^{-1}$ the latter equation becomes a " $q$ "-difference equation of the second order for the variable $x$ with " $q$ " $=p^{r}$. It is appropriate to call equation (84) the rarefied elliptic hypergeometric equation, however, 
we were not able to derive a compact form of its coefficients. Note that we know already one of its solutions given by the $V_{\epsilon}^{(r)}$-function. Its second linearly independent solution is obtained by application of the symmetry transformation of the equation which is not a symmetry of the solution, e.g. by multiplying its parameters by the powers of $q^{r}$ or some other means. The second order finite-difference equation (84) represents currently the most complicated known equation of such type with the closed form solutions (an "exactly solvable" equation).

Similar to the standard $r=1$ case, equation (82) has a partner obtained by permuting the bases $p$ and $q$ :

$$
\begin{aligned}
& \mathcal{A}\left(t_{1} p^{n_{1}+\epsilon / 2}, t_{2} p^{n_{2}+\epsilon / 2}, \ldots, t_{8} p^{n_{8}+\epsilon / 2}, q ; p^{r}\right)\left(U_{\epsilon}\left(q t_{1}, q^{-1} t_{2}, n_{1}+1, n_{2}-1\right)-U_{\epsilon}\left(t_{a}, n_{a}\right)\right) \\
& +\mathcal{A}\left(t_{2} p^{n_{2}+\epsilon / 2}, t_{1} p^{n_{1}+\epsilon / 2}, \ldots, t_{8} p^{n_{8}+\epsilon / 2}, q ; p^{r}\right)\left(U_{\epsilon}\left(q^{-1} t_{1}, q t_{2}, n_{1}-1, n_{2}+1\right)-U_{\epsilon}\left(t_{a}, n_{a}\right)\right) \\
& \quad+U_{\epsilon}\left(t_{a}, n_{a}\right)=0 .
\end{aligned}
$$

Let us set $\epsilon=0$ and denote

$$
t_{1}:=c x, \quad t_{2}:=\frac{c}{x}, \quad \text { or } \quad c=\sqrt{t_{1} t_{2}}, \quad x=\sqrt{\frac{t_{1}}{t_{2}}}
$$

and

$$
n_{1}:=n_{c}+n, \quad n_{2}:=n_{c}-n, \quad \text { or } \quad n_{c}=\frac{n_{1}+n_{2}}{2}, \quad n=\frac{n_{1}-n_{2}}{2} .
$$

Now we introduce new continuous and discrete variables

$$
\begin{gathered}
s_{1}:=\frac{c}{t_{3} p q^{1-r}}, \quad s_{2}:=\frac{c}{t_{3}}, \quad s_{3}:=c t_{3} q^{4 r}, \quad s_{a}:=\frac{p q^{1-r}}{c t_{a}}, a=4, \ldots, 8, \\
k_{1}=k_{2}:=n_{c}-n_{3}, \quad k_{3}:=n_{c}+n_{3}, \quad k_{a}:=-n_{c}-n_{a}, a=4, \ldots, 8 .
\end{gathered}
$$

To keep integrality of the numbers $k_{a}$ we shall assume that all $n_{a}$ are either odd or even. It is easy to see that the balancing condition remains intact

$$
\prod_{a=1}^{8} s_{a}=p^{2} q^{2}, \quad \sum_{a=1}^{8} k_{a}=0 .
$$

Replacing $U_{\epsilon}\left(t_{a}, n_{a}\right)$ by unknown function $f(x, n)$, we obtain another form of the rarefied elliptic hypergeometric equation:

$$
\begin{aligned}
& A\left(x q^{-n}\right)(f(p x, n-1)-f(x, n)) \\
& \quad+A\left(x^{-1} q^{n}\right)\left(f\left(p^{-1} x, n+1\right)-f(x, n)\right)+\nu f(x, n)=0,
\end{aligned}
$$

where

$$
A(x)=\frac{\prod_{a=1}^{8} \theta\left(\frac{s_{a} x}{q^{k_{a}}} ; q^{r}\right)}{\theta\left(x^{2}, p q^{1-r} x^{2} ; q^{r}\right)}, \quad \nu=\prod_{a=3}^{8} \theta\left(\frac{s_{1} s_{a}}{q^{k_{1}+k_{a}}} ; q^{r}\right)
$$




\section{EXTENSION OF THE MULTIPLE INTEGRAL SYMMETRY TRANSFORMATIONS}

We now consider rarefied analogues of the symmetry transformations for multiple elliptic hypergeometric integrals derived by Rains in [36]. Define the general type I rarefied elliptic hypergeometric function on the root system $C_{d}$ :

$$
\begin{aligned}
I_{C_{d}, \epsilon}^{(m)}\left(t_{a}, n_{a}\right) & :=\kappa_{d}^{(r)} \sum_{m_{1}, \ldots, m_{d}=0}^{r-1} \int_{\mathbb{T}^{d}} \prod_{1 \leq j<k \leq d} \frac{1}{\left.\Gamma\left(z_{j}^{ \pm 1} z_{k}^{ \pm 1}, \pm\left(m_{j}+\epsilon / 2\right) \pm\left(m_{k}+\epsilon / 2\right)\right)\right)} \\
\times & \prod_{j=1}^{n} \frac{\prod_{a=1}^{2 d+2 m+4} \Gamma\left(t_{a} z_{j}, n_{a}+m_{j}+\epsilon\right) \Gamma\left(t_{a} z_{j}^{-1}, n_{a}-m_{j}\right)}{\Gamma\left(z_{j}^{ \pm 2}, \pm\left(2 m_{j}+\epsilon\right)\right)} \frac{d z_{j}}{z_{j}}
\end{aligned}
$$

where $\left|t_{a}\right|<1$ and the balancing condition has the form

$$
\prod_{a=1}^{2 d+2 m+4} t_{a}=(p q)^{m+1}, \quad \sum_{a=1}^{2 d+2 m+4} n_{a}+(d+m+2) \epsilon=0 .
$$

Conjecture 1. Suppose that all $t_{a} \in \mathbb{C}^{*}$ and $n_{a} \in \mathbb{Z}, a=1, \ldots, 2 d+2 m+4, \epsilon=0,1$ satisfy the constraints $\sqrt{|p q|}<\left|t_{a}\right|<1$ and the balancing condition (89). Then

$$
I_{C_{d}, \epsilon}^{(m)}\left(t_{a}, n_{a}\right)=\prod_{1 \leq a<b \leq 2 d+2 m+4} \Gamma\left(t_{a} t_{b}, n_{a}+n_{b}+\epsilon\right) I_{C_{m}, \epsilon}^{(n)}\left(\frac{\sqrt{p q}}{t_{a}},-n_{a}-\epsilon\right) .
$$

For $r=1$ this is the Rains' $C_{d} \leftrightarrow C_{m}$ transformation for type I elliptic hypergeometric integrals (see Theorem 3.1 in [36]). For $m=0$ one gets evaluation of the type I integral (46). Equivalently, it can be obtained from the general formula (90) after fixing $n_{2 d+4+a}+$ $n_{2 d+4+a+m}+\epsilon=0$ and taking the limit $t_{2 d+4+a} t_{2 d+m+4+a} \rightarrow p q$ for $a=1, \ldots, m$. In this case the parameters $t_{2 d+5}, \ldots, t_{2 d+2 m+4}$ and $n_{2 d+5}, \ldots, n_{2 d+2 m+4}$ simply drop out from the expression on the left-hand side, and on the right-hand side a number of poles pinch the integration contours reducing sums of integrals to the right-hand side expression in (46). For $n=m=1$ we obtain the third $V_{\epsilon}^{(r)}$-function transformation (78). For arbitrary $r$ and $\epsilon=0$ this transformation was recently established in [32] in terms of the gamma function (62) (the mod $r$ relation in discrete balancing condition of 32] disappears after passing to our normalization of the rarefied elliptic gamma function).

Define now the type II $C_{d}$-root system analogue of the rarefied elliptic hypergeometric function (73). Take two bases $p, q \in \mathbb{C},|p|,|q|<1$, and 19 continuous and discrete parameters $t, t_{a} \in \mathbb{C}^{*}$ and $n, n_{a} \in \mathbb{Z}(a=1, \ldots, 8), \epsilon=0,1$, and impose the balancing condition

$$
t^{2 d-2} \prod_{a=1}^{8} t_{a}=(p q)^{2}, \quad 2 n(d-1)+\sum_{a=1}^{8} n_{a}+4 \epsilon=0 .
$$

The type II $C_{d^{-}}$extension of the $V$-function has the form

$$
V_{\epsilon}^{(r)}\left(t, t_{a}, n, n_{a}\right):=\kappa_{d}^{(r)} \sum_{m_{1}, \ldots, m_{r}=0}^{r-1} \int_{\mathbb{T}^{d}} \Delta_{\epsilon}^{(r)}\left(z_{k}, m_{k} ; t, t_{a}, n, n_{a}\right) \prod_{j=1}^{d} \frac{d z_{j}}{z_{j}},
$$


where

$$
\begin{gathered}
\Delta_{\epsilon}^{(r)}\left(z_{k}, m_{k} ; t, t_{a}, n, n_{a}\right)=\prod_{1 \leq j<k \leq d} \frac{\Gamma\left(t z_{j}^{ \pm 1} z_{k}^{ \pm 1}, n \pm\left(m_{j}+\frac{\epsilon}{2}\right) \pm\left(m_{k}+\frac{\epsilon}{2}\right)\right)}{\Gamma\left(z_{j}^{ \pm 1} z_{k}^{ \pm 1}, \pm\left(m_{j}+\frac{\epsilon}{2}\right) \pm\left(m_{k}+\frac{\epsilon}{2}\right)\right)} \\
\times \prod_{j=1}^{d} \frac{\prod_{a=1}^{8} \Gamma\left(t_{a} z_{j}, n_{a}+\epsilon+m_{j}\right) \Gamma\left(t_{a} z_{j}^{-1}, n_{a}-m_{j}\right)}{\Gamma\left(z_{j}^{ \pm 2}, \pm\left(2 m_{j}+\epsilon\right)\right)}
\end{gathered}
$$

with $|t|,\left|t_{a}\right|<1$. For $r=1$ this is the function introduced by Rains in [36] (see the function $I I_{\lambda, \mu}^{(n)}$ on p. 224 for $\lambda=\mu=0$ ).

Conjecture 2. The following identity should hold true

$$
\begin{gathered}
V_{\epsilon}^{(r)}\left(t, t_{a}, n, n_{a}\right)=\prod_{1 \leq a<b \leq 4} \prod_{l=0}^{d-1} \Gamma\left(t^{l} t_{a} t_{b}, l n+n_{a}+n_{b}+\epsilon\right) \\
\times \Gamma\left(t^{l} t_{a+4} t_{b+4}, l n+n_{a+4}+n_{b+4}+\epsilon\right) V_{\delta}^{(r)}\left(t, s_{a}, k_{a}\right),
\end{gathered}
$$

where

$$
\begin{gathered}
s_{a}=f t_{a}, a=1,2,3,4, \quad s_{a}=f^{-1} t_{a}, a=5,6,7,8, \quad f=\sqrt{\frac{p q t^{1-d}}{t_{1} t_{2} t_{3} t_{4}}}, \\
\begin{cases}k_{a}=n_{a}-\frac{1}{2}\left(\sum_{b=1}^{4} n_{b}+(d-1) n+\epsilon+\delta\right), & a=1,2,3,4, \\
k_{a}=n_{a}-\frac{1}{2}\left(\sum_{b=5}^{8} n_{b}+(d-1) n+\epsilon+\delta\right), & a=5,6,7,8,\end{cases}
\end{gathered}
$$

and $|t|,\left|t_{a}\right|,\left|s_{a}\right|<1$ together with the condition that $\delta$ is fixed from the demand that $\sum_{b=1}^{4} n_{b}+(d-1) n+\epsilon+\delta$ is even.

For $d=1$ this is the relation (75) proven above. For $r=1, d>1$ this is Rains' identity [36] (see Theorem 9.7 for $\lambda=\mu=0$ ). In the limit $t_{7} t_{8} \rightarrow p q$ and $n_{7}+n_{8}+\epsilon=0$ the left-hand side integral reduces to the integral in (69), whereas the right-hand side integral should collapse to the required product of gamma functions due to pinching of the integration contours.

The general rarefied elliptic hypergeometric function of type I for the root system $A_{n}$ has the form

$$
\begin{aligned}
& I_{A_{n}, \epsilon}^{(m)}\left(t_{a}, n_{a} ; s_{a}, k_{a}\right):=\frac{\left(p^{r} ; p^{r}\right)_{\infty}^{n}\left(q^{r} ; q^{r}\right)_{\infty}^{n}}{(n+1) !(2 \pi \mathrm{i})^{n}} \\
\times & \sum_{\substack{0 \leq m_{1}, \ldots, m_{n} \leq r-1 \\
m_{1}+\cdots+m_{n+1}=\epsilon}} \int_{\mathbb{T}^{n}} \frac{\prod_{j=1}^{n+1} \prod_{a=1}^{n+m+2} \Gamma\left(t_{a} z_{j}, n_{a}+m_{j}\right) \Gamma\left(s_{a} z_{j}^{-1}, k_{a}-m_{j}\right)}{\prod_{1 \leq i<j \leq n+1} \Gamma\left(z_{i} z_{j}^{-1}, m_{i}-m_{j}\right) \Gamma\left(z_{i}^{-1} z_{j},-m_{i}+m_{j}\right)} \prod_{j=1}^{n} \frac{d z_{j}}{z_{j}},
\end{aligned}
$$

where $\prod_{j=1}^{n+1} z_{j}=1,\left|t_{a}\right|,\left|s_{a}\right|<1$ and the balancing condition has the form

$$
T S=(p q)^{m+1}, \quad T:=\prod_{a=1}^{n+m+2} t_{a}, \quad S:=\prod_{a=1}^{n+m+2} s_{a}, \quad \sum_{a=1}^{n+m+2}\left(n_{a}+k_{a}\right)=0 .
$$


Suppose that $\epsilon=l(n+1)$ for some integer $l$. Then $\epsilon$ can be removed by simple shifts $m_{a} \rightarrow m_{a}+l$. Therefore the natural values of the parameter $\epsilon$, when it cannot be removed in this way, are $\epsilon=0,1, \ldots, n$. The function (95) has been considered recently in [32] in terms of the gamma function (62).

Suppose that all $t_{a}, s_{a} \in \mathbb{C}^{*}$ and $n_{a} k_{a} \in \mathbb{Z}, a=1, \ldots, n+m+2, \epsilon=0,1, \ldots, n$ satisfy the constraints $\left|t_{a}\right|,\left|T^{\frac{1}{m+1}} t_{a}^{-1}\right|,\left|s_{a}\right|,\left|S^{\frac{1}{m+1}} s_{a}^{-1}\right|<1$ and the balancing condition (96)). Then one has the following symmetry transformation

$$
\begin{aligned}
& I_{A_{n}, \epsilon}^{(m)}\left(t_{a}, n_{a} ; s_{a}, k_{a}\right)=\prod_{1 \leq a<b \leq n+m+2} \Gamma\left(t_{a} s_{b}, n_{a}+k_{b}\right) \\
& \quad \times I_{A_{m}, \delta}^{(n)}\left(\frac{T^{\frac{1}{m+1}}}{t_{a}}, N-n_{a} ; \frac{S^{\frac{1}{m+1}}}{s_{a}},-N-k_{a}\right), \quad N:=\frac{\sum_{b=1}^{n+m+2} n_{b}+\epsilon-\delta}{m+1},
\end{aligned}
$$

where the value of $\delta=0,1, \ldots, m$ is fixed from the condition that $\sum_{b=1}^{n+m+2} n_{b}+\epsilon-\delta$ is divisible by $m+1$.

For $n=m=1$ this identity coincides with the second $V_{\epsilon}^{(r)}$-function transformation (77), provided one substitutes $s_{a}:=t_{a+4}$ and $k_{a}:=n_{a+4}-\epsilon, a=1, \ldots, 4$. For $r=$ $1, n>1, m>0$ this is Rains' $A_{n} \leftrightarrow A_{m}$ transformation (see Theorem 4.1 in [36]). A symmetry transformation for $I_{A_{n}, \epsilon}^{(m)}$-function was suggested in [32, but the original proposition contained a mistake, which was corrected after the author proposed (97). The final transformation given in [32] is equivalent to (97) and has a simpler form.

As to possible proof of the above Conjecture 1, the considerations of [32] should be applicable to it as well. However, a substantially more elegant approach would consist in the appropriate generalization of the method suggested in [37. The $m=0$ case of (97) should be easily provable by direct extension of the method of [47] used there for the type I $A_{n}$ elliptic beta integral evaluation. The only available at the moment possible approach to multivariate type II $V_{\epsilon}^{(r)}$-function transformation consists in an appropriate generalization of the rather complicated proof of the $r=1$ case suggested by Rains [36]. We do not dwell into these considerations in the present work.

Next we consider possible applications of the type II $C_{n}$ rarefied elliptic hypergeometric function. For simplicity we limit ourself to the case $\epsilon=0$. Similar to the situation investigated in [46, 48, we consider the space of sequences of holomorphic functions of $z_{j} \in \mathbb{C}^{*}$, which are $r$-periodic in the discrete variables, $\varphi\left(z_{j}, \ldots, m_{k}, m_{k}+r, m_{k+1}, \ldots\right)=$ $\varphi\left(z_{j}, m_{j}\right)$, and define the inner product for it

$$
\langle\varphi, \psi\rangle=\kappa_{d}^{(r)} \sum_{m_{1}, \ldots, m_{d}=0}^{r-1} \int_{\mathbb{T}^{d}} \Delta_{0}^{(r)}\left(z_{k}, m_{k} ; t, t_{a}, n, n_{a}\right) \varphi\left(z_{j}, m_{j}\right) \psi\left(z_{j}, m_{j}\right) \prod_{k=1}^{d} \frac{d z_{k}}{z_{k}}
$$


with the weight function (93). Let us introduce the finite-difference operator

$$
\begin{aligned}
& \mathcal{D}=\sum_{j=1}^{d}\left(A_{j}\left(z_{k} q^{-m_{k}}\right)\left(T_{p, j} S_{j}^{-1}-1\right)+A_{j}\left(z_{k}^{-1} q^{m_{k}}\right)\left(T_{p, j}^{-1} S_{j}-1\right)\right), \\
& A_{j}\left(z_{k}\right)=\frac{\prod_{a=1}^{8} \theta\left(t_{a} q^{-n} z_{j} ; q^{r}\right)}{\theta\left(z_{j}^{2}, p q^{1-r} z_{j}^{2} ; q^{r}\right)} \prod_{\substack{\ell=1 \\
\ell \neq j}}^{d} \frac{\theta\left(t q^{-n} z_{j} z_{\ell}^{ \pm 1} ; q^{r}\right)}{\theta\left(z_{j} z_{\ell}^{ \pm 1} ; q^{r}\right)}
\end{aligned}
$$

where $T_{p, j}^{n} S_{j}^{m} f\left(z_{k}, m_{k}\right)=f\left(\ldots, p^{n} z_{j}, \ldots, m_{j}+m, \ldots\right)$ and we assume validity of the balancing restriction (91). For $r=1$ this is the Hamiltonian of the van Diejen completely integrable model [12] under the additional balancing condition. Suppose that the parameters $t_{a}$ are constrained in such a way that the unit circle $\mathbb{T}$ separates the sequences of poles converging to zero $z_{j}=0$ in the expression $\langle\varphi, \mathcal{D} \psi\rangle$ from their partners going to infinity. Then the operator (98) is symmetric with respect to the above inner product,

$$
\langle\varphi, \mathcal{D} \psi\rangle=\langle\mathcal{D} \varphi, \psi\rangle \text {. }
$$

Surprisingly, this statement requires a rather complicated computation associated with the presence of the powers $q^{r}$ in the arguments of theta functions. Because the suggested generalization of the van Diejen operator does not touch its analytical structure (or, more precisely, does not change the divisor structure of the functional coefficients entering it), our operator should define a completely integrable quantum many body system as well (in the sense that there exist $d$ commuting finite-difference operators of a similar form of the higher order in the shifting operators $T_{p, j} S_{j}^{-1}$ ). One can remove the balancing condition and consider a more general model, but this leads to a substantial complication of the form of the operator $\mathcal{D}$ which requires a separate consideration.

The function $f\left(z_{k}, m_{k}\right)=1$ is an evident $\lambda=0$ solution of the standard eigenvalue problem for the operator (98), $\mathcal{D} f\left(z_{k}, m_{k}\right)=\lambda f\left(z_{k}, m_{k}\right)$. The norm of this eigenfunction

$$
\langle 1,1\rangle=V_{0}^{(r)}\left(t, t_{a}, n, n_{a} ; p, q\right)
$$

is exactly the type II multivariable analogue of the rarefied elliptic hypergeometric function for the root system $C_{d}$ described above.

Another application to eigenvalue problems comes from comparing the operator $\mathcal{D}$ with the rarefied elliptic hypergeometric equation in the form (86). One can see that the latter equation represents the eigenvalue problem for the operator $\mathcal{D}$ with the following three special restrictions: 1) $\left.d=1,2) t_{2}=t_{1} p q^{1-r}, 3\right) \lambda=-\nu$, i.e. the $d=1$ function $V_{\epsilon=0}$ is now interpreted as an eigenfunction of $\mathcal{D}$ with an additional restriction on the parameters and a particular eigenvalue. This is completely similar to the situation taking place in the $r=1$ case [46, 48].

\section{Conclusion}

In this paper we have proved several identities for the rarefied elliptic hypergeometric functions and formulated a few related conjectures. Summarizing them it is natural to 
expect that all exact relations either proven [9, 36, 49] or conjectured [50, 51] have rarefied analogues obtained simply by replacing the elliptic gamma functions $\Gamma(z ; p, q)$ to $\Gamma^{(r)}(z, m ; p, q)$ and integrations $\int_{\mathbb{T}^{d}}$ to $\sum_{m_{1}, \ldots, m_{d}=0}^{r-1} \int_{\mathbb{T}^{d}}$. If true, this yields a tremendous amount of new handbook formulae. Furthermore, one can consider their various degeneration limits enlarging further the number of exact formulae. Indeed, the elliptic hypergeometric integrals can be reduced to hyperbolic integrals [29, 35], which corresponds to the reduction of $4 d$ superconformal indices to $3 d$ partition functions [38. Applying a similar limit to the rarefied versions of these integrals, one gets the rarefied hyperbolic integrals, or $3 d$ partition functions on the squashed lens space, which was mentioned already in [7]. Some of such functions were considered recently in [23].

As we have shown, the $p \rightarrow 0$ limit of the rarefied elliptic hypergeometric functions lead to new $q$-hypergeometric identities requiring proper systematic investigation. One can degenerate our sums of integrals to terminating rarefied elliptic hypergeometric series and consider their $p \rightarrow 0$ degenerations as well.

Let us shift the discrete summation variables $m_{\ell} \rightarrow m_{\ell}-[r / 2]$, where $[x]$ is the integral part of the real variable $x$, and take the limit $r \rightarrow \infty$. Such a limit describes a degeneration of superconformal indices of four-dimensional theories on $S^{1} \times L(r,-1)$ to superconformal indices of three-dimensional field theories on the squashed three sphere [7]. Again, this yields a very large number of exact identities for corresponding infinite bilateral sums of $q$-hypergeometric integrals, similar to the simplest case considered in [31]. As shown in [3], partition functions of $4 d$ supersymmetric field theories on $S^{1} \times S^{3}$ space-time are equal to the corresponding superconformal indices up to an exponential of the Casimir energy. It is natural to expect that similar situation holds for partition functions on lens spaces and the rarefied elliptic hypergeometric functions.

The curious discrete variable $\epsilon$ emerging for $r>1$ deforms various symmetries of the original elliptic hypergeometric integrals. It breaks the $S U(n)$ (or $S p(2 n)$ ) gauge symmetry by mixing with a global $U(1)$ group which acts nontrivially only on the lens space holonomies. It plays also an important role in the definition of the action of full $W\left(E_{7}\right)$ group on the vectors from the discrete space $\mathbb{Z}^{8}$ and related reflections acting in the space $\mathbb{Z}^{2 n+2 m+4}$. So, a proper interpretation of the discrete variable $\epsilon$ in terms of the lens space superconformal indices is one of interesting physical questions.

The integrals considered in this work are related only to the simplest lens space $L(r,-1)$. It is possible to extend them to the general lens space $L(r, k)$, which adds more discrete parameters. As to other applications of our results, let us mention that it is not difficult to formulate a generalization of the Bailey lemma [45] on the basis on the rarefied elliptic beta integral and use it for deriving a solution of the Yang-Baxter equation that extends the $R$-operator of [11] to $r>1$ (this problem is related to the $2 d$ integrable lattice model of [31 and corresponding star-triangle relation). The same technique can be used for generalization of the elliptic hypergeometric integral identities used in the $2 d$ topological field theories [38] together with many other results for standard elliptic hypergeometric functions 49. 
Acknowledgements. This work is supported by the Russian Science Foundation (project no. 14-11-00598). The author is indebted to A. P. Kels for explaining an equivalence of the $\epsilon=0$ rarefied elliptic beta integral evaluation considered in this paper to the one of [31], as well as an equivalence of the balancing conditions in [32] involving mod $r$ relation to ours after appropriate adjustment of discrete parameters. The referees are thanked for constructive remarks and suggestions. A part of this work was done during a visit to the Max Planck Institute for Mathematics in Bonn and the author is indebted to this institute for a kind hospitality.

\section{REFERENCES}

[1] V. P. Alekseevksi, On functions similar to the function gamma, Proceedings of the Kharkov Math. Society, (2) 1 (1889), 169-238 (in Russian).

[2] G. E. Andrews, R. Askey, and R. Roy, Special Functions, Encyclopedia of Math. Appl. 71, Cambridge Univ. Press, Cambridge, 1999.

[3] B. Assel, D. Cassani, and D. Martelli, Localization on Hopf surfaces, J. High Energy Phys. 1408 (2014), 123.

[4] E. W. Barnes, On the theory of the multiple gamma function, Trans. Cambridge Phil. Soc. 19 (1904), 374-425.

[5] R. J. Baxter, Partition function of the eight-vertex lattice model, Ann. Phys. 70 (1972), 193-228.

[6] V. V. Bazhanov, V. V. Mangazeev, and S. M. Sergeev, Exact solution of the Faddeev-Volkov model, Phys. Lett. A 372 (2008), 1547-1550.

[7] F. Benini, T. Nishioka, and M. Yamazaki, $4 d$ Index to $3 d$ index and $2 d T F Q T$, Phys. Rev. D 86 (2012), 065015.

[8] F. J. van de Bult, An elliptic hypergeometric beta integral transformation, arXiv:0912.3812.

[9] - Two multivariate quadratic transformations of elliptic hypergeometric integrals, arXiv:1109.1123

[10] E. Date, M. Jimbo, A. Kuniba, T. Miwa, and M. Okado, Exactly solvable SOS models, II: Proof of the star-triangle relation and combinatorial identities, Adv. Stud. in Pure Math. 16 (1988), $17-122$.

[11] S. E. Derkachov and V. P. Spiridonov, Yang-Baxter equation, parameter permutations, and the elliptic beta integral, Uspekhi Mat. Nauk 68 (6) (2013), 59-106 (Russian Math. Surveys 68 (6) (2013), 1027-1072).

[12] J. F. van Diejen, Integrability of difference Calogero-Moser systems, J. Math. Phys. 35 (1994), 2983-3004.

[13] J. F. van Diejen and V. P. Spiridonov, An elliptic Macdonald-Morris conjecture and multiple modular hypergeometric sums, Math. Res. Letters 7 (2000), 729-746.

[14] _ Elliptic Selberg integrals, Internat. Math. Res. Notices, no. 20 (2001), 1083-1110.

[15] T. Dimofte and D. Gaiotto, An $E_{7}$ surprise, J. High Energy Phys. 10 (2012), 129.

[16] A. L. Dixon, On a generalization of Legendre's formula $K E^{\prime}-(K-E) K^{\prime}=\pi / 2$, Proc. London Math. Soc. (2), 3 (1) (1905), 206-224.

[17] F. A. Dolan and H. Osborn, Applications of the superconformal index for protected operators and q-hypergeometric identities to $\mathcal{N}=1$ dual theories, Nucl. Phys. B 818 (2009), 137-178.

[18] G. Felder and A. Varchenko, The elliptic gamma function and $S L(3, \mathbb{Z}) \ltimes \mathbb{Z}^{3}$, Adv. Math. 156 (2000), 44-76.

[19] P. J. Forrester and S. O. Warnaar, The importance of the Selberg integral, Bull. Amer. Math. Soc. 45 (2008), 489-534. 
[20] I. B. Frenkel and V. G. Turaev, Elliptic solutions of the Yang-Baxter equation and modular hypergeometric functions. The Arnold-Gelfand mathematical seminars, Birkhäuser Boston, Boston, MA, 1997, pp. 171-204.

[21] E. Friedman and S. Ruijsenaars, Shintani-Barnes zeta and gamma functions, Adv. Math. 187 (2004), 362-395.

[22] A. Gadde, E. Pomoni, L. Rastelli, and S. S. Razamat, S-duality and $2 d$ topological QFT, J. High Energy Phys. 03 (2010), 032.

[23] I. Gahramanov and A. P. Kels, The star-triangle relation, lens partition function, and hypergeometric sum/integrals, J. High Energ. Phys. 02 (2017), 040.

[24] D. Gaiotto and H.-C. Kim, Duality walls and defects in $5 d \mathcal{N}=1$ theories, J. High Energy Phys. 01 (2017), 019.

[25] G. Gasper and M. Rahman, Basic Hypergeometric Series, Encyclopedia of Math. Appl. 96, Cambridge Univ. Press, Cambridge, 2004.

[26] R. A. Gustafson, A generalization of Selberg's beta integral, Bull. Amer. Math. Soc. (N.S.) 22 (1990), 97-105.

[27] _ Some q-beta integrals on $S U(n)$ and $S p(n)$ that generalize the Askey-Wilson and Nassrallah-Rahman integrals, SIAM J. Math. Anal. 25 (1994), 441-449.

[28] F. H. Jackson, The basic gamma-function and the elliptic functions, Proc. Roy. Soc. London A 76 (1905), 127-144.

[29] M. Jimbo and T. Miwa, Quantum KZ equation with $|q|=1$ and correlation functions of the XXZ model in the gapless regime, J. Phys. A: Math. Gen. 29 (1996), 2923-2958.

[30] A. P. Kels, A new solution of the star-triangle relation, J. Phys. A: Math. Theor. 47 (2014), 055203.

[31] _ New solutions of the star-triangle relation with discrete and continuous spin variables, J. Phys. A: Math. \& Theor. 48 (2015), 435201.

[32] A. P. Kels and M. Yamazaki, Elliptic hypergeometric sum/integral transformations and supersymmetric lens index, arXiv:1704.03159v1; SIGMA 14 (2018), 013.

[33] M. A. Naimark, Decomposition of a tensor product of irreducible representations of the proper Lorentz group into irreducible representations, Tr. Mosk. Mat. Obs. 8 (1959), 121-153 (Am. Math. Soc. Transl., Ser. 2, Vol. 36 (1964), 101-229).

[34] M. Rahman, An integral representation of $a_{10} \phi_{9}$ and continuous bi-orthogonal ${ }_{10} \phi_{9}$ rational functions, Can. J. Math. 38 (1986), 605-618.

[35] E. M. Rains, Limits of elliptic hypergeometric integrals, Ramanujan J. 18 (3) (2009), 257-306.

[36] _ , Transformations of elliptic hypergeometric integrals, Ann. of Math. 171 (2010), 169243.

[37] E. M. Rains and V. P. Spiridonov, Determinants of elliptic hypergeometric integrals, Funkt. Analiz i ego Pril. 43 (4) (2009), 67-86 (Funct. Analysis and its Appl. 43 (4) (2009), 297-311).

[38] L. Rastelli and S. S. Razamat, The supersymmetric index in four dimensions, J. Phys. A: Math. and Theor. 50 (2017), 443013.

[39] S. S. Razamat and B. Willett, Global properties of supersymmetric theories and the lens space, Commun. Math. Phys. 334 (2015), 661-696.

[40] H. Rosengren, Elliptic hypergeometric functions, Lectures at OPSF-S6, College Park, Maryland, 11-15 July 2016, arXiv:1608.06161.

[41] S. N. M. Ruijsenaars, First order analytic difference equations and integrable quantum systems, J. Math. Phys. 38 (1997), 1069-1146.

[42] A. Selberg, Bemerkninger om etmultipelt integral, Norsk Mat. Tiddskr. 26 (1948), 71-78 (English translation in Collected Papers, Vol. I, Springer, Berlin, 1989, 204-213).

[43] V. P. Spiridonov, On the elliptic beta function, Uspekhi Mat. Nauk 56 (1) (2001), 181-182 (Russ. Math. Surveys 56 (1) (2001), 185-186). 
[44] _ Theta hypergeometric integrals, Algebra i Analiz 15 (6) (2003), 161-215 (St. Petersburg Math. J. 15 (6) (2004), 929-967).

[45] _ A Bailey tree for integrals, Teor. Mat. Fiz. 139 (1) (2004), 104-111 (Theor. Math. Phys. 139 (1) (2004), 536-541).

[46] _ Elliptic hypergeometric functions, Habilitation thesis, Joint Institute for Nuclear Research, Dubna, September 2004, 218 pp., arXiv:1610.01557.

[47] _ Short proofs of the elliptic beta integrals, Ramanujan J. 13 (1-3) (2007), 265-283.

[48] __ Elliptic hypergeometric functions and Calogero-Sutherland type models, Teor. Mat. Fiz, 150 (2) (2007), 311-324 (Theor. Math. Phys. 150 (2) (2007), 266-277).

[49] _ Essays on the theory of elliptic hypergeometric functions, Uspekhi Mat. Nauk 63 (3) (2008), 3-72 (Russian Math. Surveys 63 (3) (2008), 405-472).

[50] V. P. Spiridonov and G. S. Vartanov, Elliptic hypergeometry of supersymmetric dualities, Commun. Math. Phys. 304 (2011), 797-874.

[51] _ Elliptic hypergeometry of supersymmetric dualities II. Orthogonal groups, knots, and vortices, Commun. Math. Phys. 325 (2014), 421-486.

[52] — Elliptic hypergeometric integrals and 't Hooft anomaly matching conditions, J. High Energy Phys. 06 (2012), 016.

[53] V. P. Spiridonov and S. O. Warnaar, Inversions of integral operators and elliptic beta integrals on root systems, Adv. in Math. 207 (2006), 91-132.

[54] V. P. Spiridonov and A. S. Zhedanov, Spectral transformation chains and some new biorthogonal rational functions, Commun. Math. Phys. 210 (2000), 49-83.

Bogoliubov laboratory of Theoretical Physics, JinR, Dubna, Moscow Region, 141980 Russia and St. Petersburg Department of the Steklov Mathematical Institute of Russian Academy of Sciences, Fontanka 27, St. Petersburg, 191023 Russia e-mail: SpiriDON@THEOR.JINR.RU 